University of Texas at El Paso

\title{
DigitalCommons@UTEP
}

Open Access Theses \& Dissertations

2018-01-01

\section{An Efficient Method For Online Identification Of Steady State For Multivariate System}

Honglun None $\mathrm{Xu}$

University of Texas at El Paso, xuhonglun38324@gmail.com

Follow this and additional works at: https://digitalcommons.utep.edu/open_etd

Part of the Computer Engineering Commons, Mathematics Commons, and the Statistics and Probability Commons

\section{Recommended Citation}

Xu, Honglun None, "An Efficient Method For Online Identification Of Steady State For Multivariate System" (2018). Open Access Theses \& Dissertations. 190.

https://digitalcommons.utep.edu/open_etd/190

This is brought to you for free and open access by DigitalCommons@UTEP. It has been accepted for inclusion in Open Access Theses \& Dissertations by an authorized administrator of DigitalCommons@UTEP. For more information, please contact lweber@utep.edu. 


\section{AN EFFICIENT METHOD FOR ONLINE IDENTIFICATION OF STEADY STATE FOR MULTIVARIATE SYSTEM}

\section{HONGLUN XU}

Master's Program in Computational Science Program

APPROVED:

Bill Tseng, Ph.D., Chair

Jianguo Wu, Ph.D., Co-Chair

Paras Mandal, Ph.D.

Amy Wagler, Ph.D.

Charles Ambler, Ph.D.

Dean of the Graduate School 
Copyright (C)

by

Honglun $\mathrm{Xu}$

2018 


\title{
AN EFFICIENT METHOD FOR ONLINE IDENTIFICATION OF STEADY STATE FOR MULTIVARIATE SYSTEM
}

\author{
by \\ HONGLUN XU, MSc
}

\begin{abstract}
THESIS
Presented to the Faculty of the Graduate School of The University of Texas at El Paso

in Partial Fulfillment

of the Requirements

for the Degree of
\end{abstract}

MASTER OF SCIENCE

Computational Science Program

THE UNIVERSITY OF TEXAS AT EL PASO

December 2018 


\section{Acknowledgements}

The research presented in this M.S thesis benefited from the valuable insights and support of many people. It is my pleasure to express my sincere gratitude to all of them.

First, I would like to express my deepest appreciation to my master advisor, Prof. Bill Tseng for his outstanding academic guidance and full support in my UTEP life. Words cannot express my sincere gratitude for his help in academic research. I am so lucky to benefit from not only his expertise in academic research, but also his great personality in all other aspects. It is impossible that my research study would have been so smooth without his encouragement and guidance.

Then, I also would like to appreciate my committee co-chair Prof. Jianguo Wu. I am so lucky to get his supervision. I have got a lot from his easy-going personality, his energetic attitude towards research and his full support in my research. I am also affected deeply by his academic attitude and the substance of a genius: he convincingly conveyed a spirit of adventure in scientific research. He always gives me many innovated ways and suggestion to help me out when I was struggling in the scientific research.

Additionally, I would be grateful to my master committee members Prof. Amy Wagler and Prof. Paras Mandal for serving on my committee and providing helpful ideas to improve my dissertation. Meanwhile, I would like to thank Prof. Ming-Ying Leung from Computational Science Program for serving on my director in my qualified exam.

My thanks also go to my friends in my research group: Dr. Aditya, Akundi, Dr. Hoejin Kim, Yuxin Wen, Dr. Zhonghua Hu, Dr. Carlos A Garcia Rosales, Anabel Renteria Marquez, Dr. Ivan Renteria Marquez and Md Fashiar Rahman. Thank you for being supportive and accompanying me in my Master studies. You really make my life in UTEP enjoyable and memorable. I also thank to my roommates in El Paso: Mr. Chunkai Shi, Ms. Xianhui Xiang, Mr. Yi Xie, Mr. Zhu and Ms. Xianxian Li. During my El Paso life, they give me a lot of help and support so that I can focus on my study and research. 
Lastly and most importantly, I want to express my special thanks to my parents for their unconditional love, support and encouragement. Without my parents' support and loving care, I would not have got this achievement in my life. Whether I have good time or tough time, they provide me support and endless love to realize my own dream with no hesitation. I am very happy to have them as my parents and so proud of it. 


\begin{abstract}
Most of the existing steady state detection approaches are designed for univariate signals. For multivariate signals, the univariate approach is often applied to each process variable and the system is claimed to be steady once all signals are steady, which is computationally inefficient and also not accurate. The article proposes an efficient online method for multivariate steady state detection. It estimates the covariance matrices using two different approaches, namely, the meansquared-deviation and mean-squared-successive-difference.

To avoid the usage of a moving window, the process means and the two covariance matrices are calculated recursively through exponentially weighted moving average. A likelihood ratio test is developed to compare the difference of the two covariance matrices and to detect the steady state. The intensive numerical studies and real case study show that the proposed method can accurately detect the steady state of a multivariate system.
\end{abstract}




\section{Table of Contents}

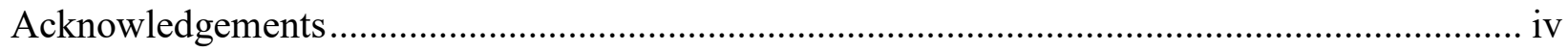

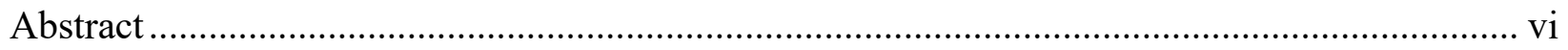

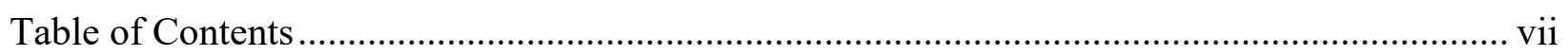

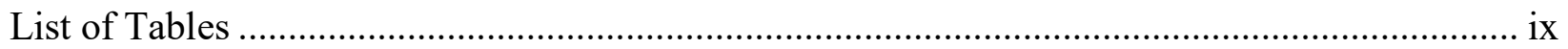

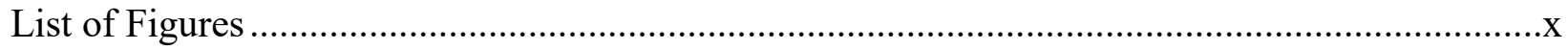

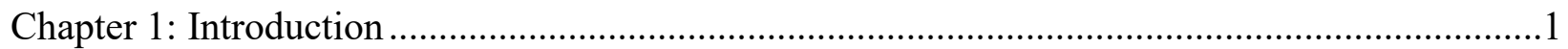

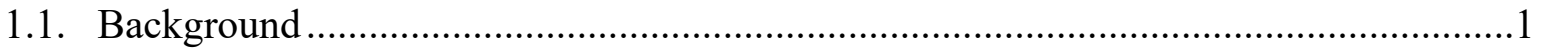

1.2. Research Objectives .........................................................................................2

1.3. Statement Problem and Rationale of the Study ....................................................... 3

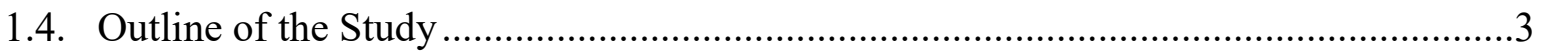

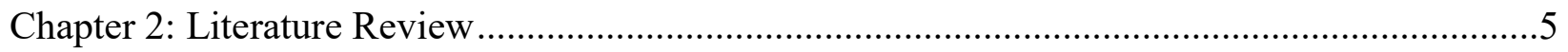

2.1. Offline Steady State Detection..........................................................................5

2.1.1. Marginal Standard Error Rules ..............................................................5

2.2. Online Steady State Detection ......................................................................6

2.2.1. Linear Regression Method ....................................................................

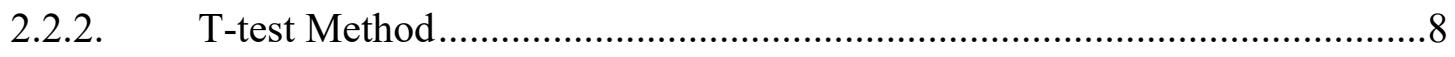

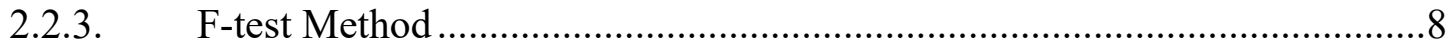

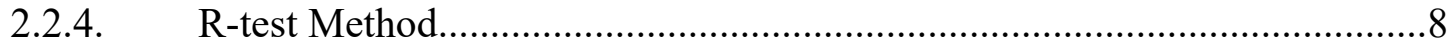

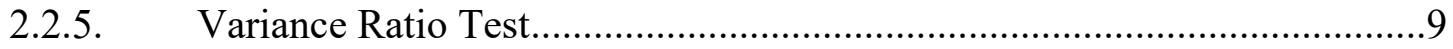

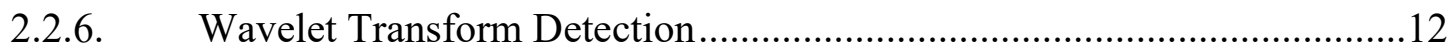

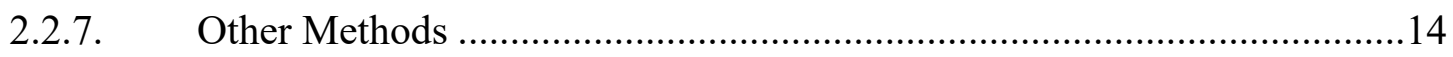

Chapter 3: A EWMA Based LRT for Multivariate Steady State Detection ...............................17

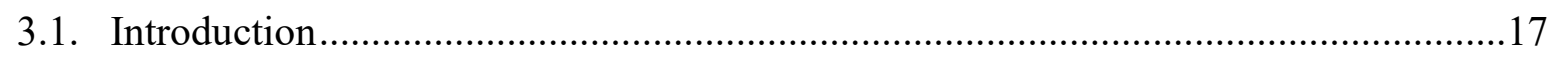

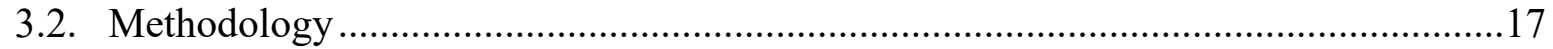

3.3. Illustration of the EWMA-LRT Method ….........................................................20

3.4. Performance Evaluation and Comparison ......................................................21

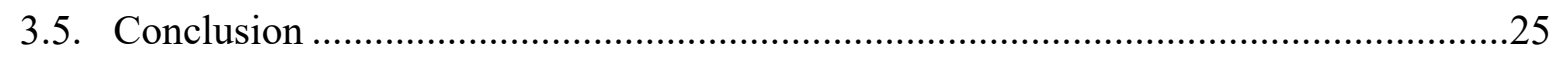

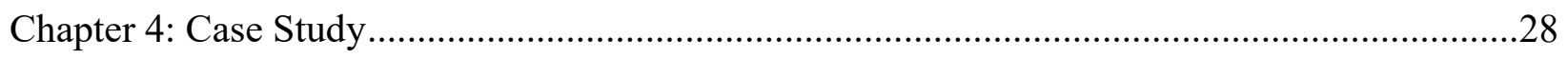

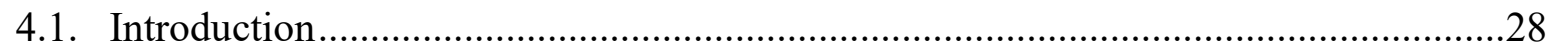


4.2. Application to the Micro/nanoparticle Dispersion Process ...................................28

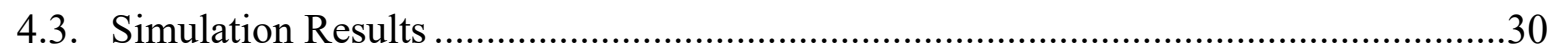

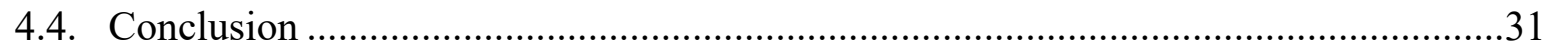

Chapter 5: Conclusion and Recommendation for Future Work ............................................32

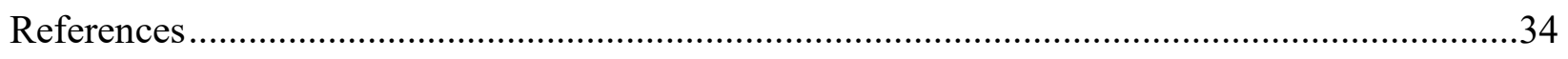

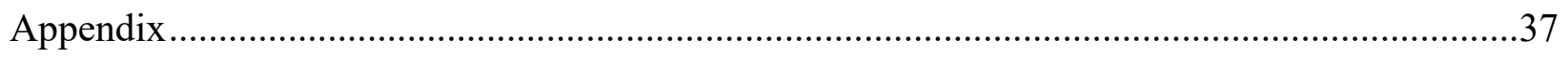

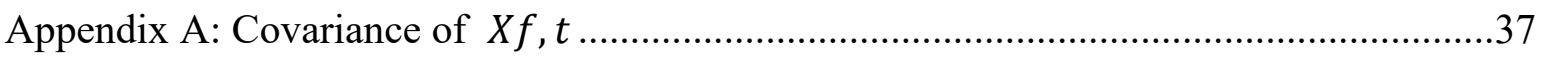

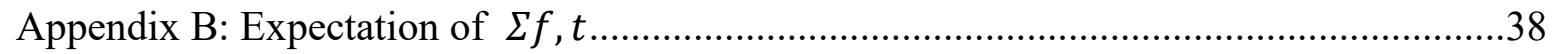

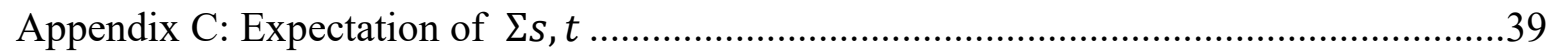

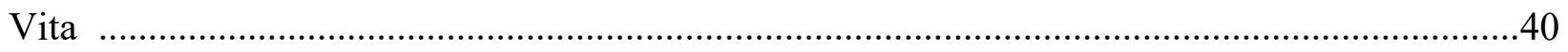




\section{List of Tables}

Table 3.1: Four Mean Bias Functions and Their Shapes ................................................... 21

Table 3.2: Noise Types and Their Parameters ..................................................................... 23

Table 3.3: Detailed Comparison of EWMA-LRT, VRT, SSD and WTD for the Case of $p=4, w \equiv$

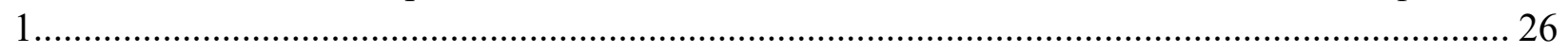

Table 3.4: Detailed Comparison of EWMA-LRT, VRT, SSD and WTD for the Case of $p=8, w \equiv$

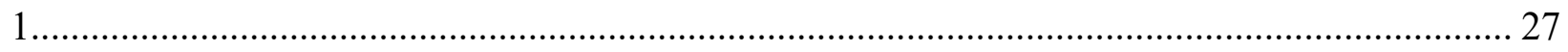




\section{List of Figures}

Figure 3.1: Illustration of the steady state detection of EWMA-LRT: (a) $T_{0}=200$, the detected steady state time $T^{*}=205$, (b) $T_{0}=300$, the detected steady-state time $T^{*}=288$. The vertical dashed line indicates the detected steady state time.

Figure 3.2: The WSE of EWMA-LRT, VRT, SSD and WTD method as a function of penalty weight $w$ : top row $(\mathrm{a}, \mathrm{b}, \mathrm{c}): p=4$; bottom row $(\mathrm{d}, \mathrm{e}, \mathrm{f}): p=8 ;(\mathrm{a}, \mathrm{d}): \operatorname{AR}(0),(\mathrm{b}, \mathrm{e}): \operatorname{AR}(1)$ and $(\mathrm{c}, \mathrm{f})$ : $\operatorname{AR}(2)$.

Figure 4.1: Ultrasonic dispersion of nanoparticles

29

Figure 4.2: Steady state detection of cavitation noise signals in the dispersion of $30 \mathrm{~g} \mathrm{Al}_{2} \mathrm{O}_{3}$ in water with ultrasonic power $40 \mathrm{~W}$. 


\section{Chapter 1: Introduction}

\subsection{Background}

Industrial and manufacturing processes includes usually noise and non-stable in nature. Due to many reasons like measurement errors, environmental effects and maintenance and so on, the process when stable, could be confounded by the noise. Detecting if a system or a process is steady is a significantly important task in many engineering problems, such as process analysis and optimization [1-4], fault detection and diagnosis [5, 6], data reconciliation [7, 8], process automation and control [9-11].

In these applications, the steady state is often defined as a state where the mean of the time series stays unchanging. The steady state detection applies statistical methods to identify or monitor the change from the non-stable state to the steady state to facilitate the optimization, performance evaluation and process control, etc. Detecting the steady state is different with the traditional statistical process control (SPC) which usually uses the control chart to detect the change from the normal condition (stable state) to the abnormal condition (non-stable state).

For example, in the chemical industry, process data (e.g., flow rate, $\mathrm{pH}$ values, temperature etc.) have to be collected under steady-state conditions for unit design, pinch analysis, process optimization, and fault detection [12]. Besides, almost all the fault detection and diagnostic (FDD) methodologies are based on steady state models and require that the system is steady $[6,13]$. Another example is batch process or batch manufacturing. During batch-to-batch start-up periods, the batch operations are not steady and cannot guarantee the product quality because the materials are not mixed well and the machine conditions such as temperature and pressure are not stable [14, 15]. Therefore, an efficient method for steady-state detection could help achieve satisfied products and avoid expensive quality inspection.

In process automation and control, the steady state can be used to trigger the next action. For example, in ultrasonic cavitation based particle dispersion process, the steady state condition of the cavitation noise has been used as a process completion indicator [11]. Once the noise signal 
is steady state, the dispersion process is considered completed and the process can be stopped. In the numerical iterative methods, such as nonlinear regression, optimization and neural network training, the steady state condition can also be used as a stopping or convergence criterion. The iterative procedures will be stopped once the objective function like the sum of squared error reaches a steady state $[9,16]$.

Engineers often conduct a series of experiments in a variety of operating conditions for data collection and process analysis. When the worker observes that stable conditions are met, each sampling event is started and the worker implements the innovative set of operational conditions. But this visual approach of detection needs repeated human attention and is subdue to human mistake in identifying detection because of noise. Meanwhile, there are factors can affect the visual judgement, which are change-of-shift timing, slow process changes and noisy measurements.

In addition, scheduling time can be carried out to create new conditions of operation for the experiments. Unfortunately, if the experiment runs are planned for an needlessly long time, this technique cannot be efficiency. If the scheduled time is not sufficient for any conditions to get the steady state, these data cannot be worth. It is not almost possible to predict the essential holding time due to the time reaching steady state varies with different operating conditions.

As a result, real-time or automated online steady state detection can be valuable to cause the next step of process phase or an experimental plan. Based on the steady state identification techniques, statistical methods are used in this study since they may be more robust for analyzing noisy process signals.

\subsection{Research Objectives}

In the last few decades, detection of steady state is more and more important for many areas such as industrial and manufacturing processes. There are more than forty offline steady-state detection algorithms developed for univariate signals. These offline methods can efficiently identify the steady-state period if the entire signal is available. Nevertheless, they are not suitable 
for real-time or online applications. The online steady state detection is based on the observations up to the current time and is more difficult.

All the aforementioned methods are for univariate systems. However, almost all systems are multivariate inherently. To guarantee that the system is steady, all the signals of the system have to be steady. Therefore, steady state detection of multivariate signals are desirable. To our best knowledge, there is a very limited number of multivariate steady state detection algorithms in the existing literature.

In this study, we have two objectives as following:

(1) A new and efficient method is developed for online steady state detection of the multivariate systems. It is an multivariate extension of Cao's univariate method [9]. Similar to the $F$-test or variance ratio test, the covariance of the multivariate signals are calculated by using two different ways, namely, the mean squared deviation and the mean squared successive difference.

(2) Then, the likelihood ratio test of two covariances is used. To make the method more robust to all signals, the filter factor method again is used to deal with the processed data.

\subsection{Statement Problem and Rationale of the Study}

Based on the background, the offline methods can identify the steady-state period if the full or entire signal is available. However, they are not suitable for real-time or online applications. The online steady state detection is based on the observations up to the current time and is more challenging. Thus, online steady state detection is difficult to be developed. Now there are several online steady-state methods, developed for univariate signals. Meanwhile, most of these online methods are developed for univariate signal and use the moving data window so that they are not robust and suitable for multivariate system.

However, developing the efficient method for online identification of steady state for multivariate systems is very significant in many areas.

\subsection{Outline of the Study}

The rest of this dissertation is organized as follows. 
Chapter 2 explains a detailed literature review of the existing steady state detection methods including the off-line detection and on-line detection. For example, Linear Regression method, F-test method, Variance Ratio Test and Wavelet Transform Detection and so on.

In Chapter 3, a robust steady state detection algorithm is developed. The approximated formula for the expectation and covariances of a multivariable systems model will be derived for the steady-state detection, computational issues will be addressed, and the virtues of the proposed identification method of the steady state is also introduced. Chapter 3 presents the numerical analysis and the selection of filter factors. Meanwhile, the comparison with several existing methods also will be demonstrated in this chapter.

Chapter 4 will give the real case study using the proposed method in the micro/nanoparticle dispersion process. The cavitation noise signals are obtained from the micro/nanoparticle dispersion process. It is found that when the cavitation noise signals enter into steady state, the dispersion process is finished.

The results and conclusion are given in Chapter 5. 


\section{Chapter 2: Literature Review}

The steady state detection applies statistical techniques to identify the transition from nonstationary state (also called transient state, start-up or warm-up period) to the steady state, which is opposite to the traditional statistical process control techniques in that it utilize control charts to monitor the change from steady state to other steady state or nonstationary state.

\subsection{Offline Steady State Detection}

In the last few decades, there are more than forty offline steady-state detection algorithms developed for univariate signals, mostly of which are developed to remove the transient period in discrete-event simulations [17]. According to Robinson and Davies [18, 19], these methods can be classified into five categories: (1) graphical methods, such as time-series inspection [20], CUSUM plots method [21], exponentially weighted moving average method (EWMA) [22], and statistical process control method [19]; (2) heuristic approaches (e.g. the marginal standard error rules [23, 24]) which offer some simple and straightforward rules to truncate the time-series data. These methods are very popular in various areas because they are not as subjective as the graphical methods; (3) statistical methods including the goodness-of-fit test [25] and wavelet-based spectral algorithms [26]; (4) initialization bias tests are to test whether there is initialization bias in the data. The representative methods include batch-means-based tests, t-tests and compound test method [27]; and (5) hybrid methods which combine the initialization bias tests and graphical or heuristic approaches to determine the warm-up period [28].

\subsubsection{Marginal Standard Error Rules}

The Marginal Standard Error Rules (MSER) [23] determines the truncation point (steady state point in this research) which minimizes the marginal confidence interval's the width about the mean of truncated sample (steady state mean). It is better than heuristic methods on models which include exponential shift bias.

This MSER algorithm is explained as follows. Shown the observations $\left\{X_{i}: i=\right.$ $1,2,3 \ldots, n\}$, and the samples of the steady state are $\left\{X_{i}: i=d+1, d+2, d+3 \ldots, n\right\}$. Then the 
$100(1-\alpha) \%$ confidence interval's the half-width for the estimate of the steady-state mean is given by

$$
C I(d+1, n)=\frac{z_{\alpha / 2} S_{n, d}}{\sqrt{n-d}}
$$

Where $z_{\alpha / 2}$ is the inverse of the cumulative density function (CDF) at the probability $(1-\alpha / 2)$ for the standard normal distribution, Then, $S_{n, d}$ is the standard sample deviation shown by

$$
S_{n, d}=\sqrt{\frac{1}{n-d-1} \sum_{i=d+1}^{n}\left(X_{i}-\bar{X}_{n, d}\right)^{2}}
$$

Where $\bar{X}_{n, d}=\frac{1}{n-d} \sum_{i=d+1}^{n} X_{i}$. Therefore, the best truncation point $d^{*}$ which minimizes the confidence interval is given as

$$
\begin{gathered}
d^{*}=\underset{n \gg d \gg 0}{\arg \min }(C I(d+1, n)) \\
=\underset{n \gg d \gg 0}{\arg \min }\left(C I(d+1, n)^{2}\right) \\
=\underset{n \gg d \gg 0}{\arg \min }\left(\frac{\sum_{i=d+1}^{n}\left(X_{i}-\bar{X}_{n, d}\right)^{2}}{(n-d-1)(n-d)}\right)
\end{gathered}
$$

Due to $\mathrm{n} \gg \mathrm{d}$, the denominator of Eq. (2.3) is simplified from $(n-d-1)(n-d)$ to $(n-d)^{2}$. Thus, this method is called as "MSER" which is given by

$$
\operatorname{MSER}=\frac{1}{(n-d)^{2}} \sum_{i=d+1}^{n}\left(X_{i}-\bar{X}_{n, d}\right)^{2}
$$

\subsection{Online Steady State Detection}

These offline methods can efficiently identify the steady-state period if the full or entire signal is available. Nevertheless, they are not suitable for real-time or online applications. The 
online steady state detection is based on the observations up to the current time and is more difficult $[2,3]$. In the existing literature, there are only several online steady-state methods, which can be summarized as follows: (1) a linear regression is performed over a moving data window, and the fitted slope is monitored. If the absolute value of the fitted slope is larger than a threshold or the

fitted line is not sufficiently "flat", the process signal is considered not steady $[11,29,30]$; (2) $t$ test is applied to compare the means of two recently obtained adjacent moving data windows. When there is no significant difference between them, the process is considered steady [31]; (3) the standard deviation of a moving data window is monitored. The steady state is detected when the standard deviation is below a threshold [6]; (4) an F-test (also called variance ratio test) is performed on the ratio of two variances of a moving window calculated by two different methods. One is the mean squared deviation, while the other one is the mean of squared differences of successive data [32]. When the process is at the steady state, the ratio is close to unity [9].

However, these methods are not robust due to the usage of a moving window [11, 33, 34]. Too long a moving window may delay the detection while too short a moving window may result in high false alarm rate. Now these methods will be introduced separately.

\subsubsection{Linear Regression Method}

The technique of linear regression is a direct method to detect steady state. The approach involves performing a data sequence's the linear regression and the evaluation of the linear regression line's slope. When the slope is close to zero, the process is likely to be in a steady state. On the other hand, the slope differs considerably from zero, the process may be in transient condition [29]. If this method were to be used online during the process, user expertise and great storage would be required to determine the length of the holding window.

For example, For the oscillating response, the slope of the linear regression is zero, which could result in incorrect stable state detection. Since human judgment is required when selecting the length of the data window, this technique is not automated. This method has also 
computationally burdensome, because the entire data window needs to be updated at every interval.

\subsubsection{T-test Method}

The T-test method is the evaluation of the mean and standard deviation value in successive $\mathrm{N}$ sample data's windows and compares the two values successively by T-statistics, which is different in averages divided by average's standard error. When the system is in stable condition, the averages are ideally the same. If the process variation is high, the T-statistical value is higher than the critical value to assert that the process is in a nonstationary state. However, the method is computationally expensive, since the means and the standard deviation must be compared and calculated at every moment.

\subsubsection{F-test Method}

The F-test technique [32] is to calculate the variance ratio that is measured from the same data's sequence by two different approaches. One variance is the mean square deviation in the window of selected data. Another variance is the mean of squared differences of successive data that is from the same data window. The ratio of two variances in a stable state process could ideally be unity. The actual ratio, however, is not exactly uniform due to random noise in the real process. The ratio may be steady-state unity.

The F-test approach is valid but has some unwanted characteristics. For example, many data must also be stored and calculated by this method, which also makes it computationally expensive. In addition, this technique needs user expertise in selecting a suitable time interval to remove autocorrelation.

\subsubsection{R-test Method}

Based on the $F$-test method, an efficient method without moving windows is developed by Cao [9], where exponentially weighted moving average (EWMA) is performed to calculate the two variances respectively. This approach estimates the variance using two different approaches, namely, the mean squared deviation and the mean squared successive difference. In order to avoid 
the usage of a moving window, the process means and the two variances are calculated respectively through exponentially weighted moving average (EWMA). It also can be extended to a multivariate process. The information of R-test method is explained in detail in the next part. This study shows that R-statistic technique is applied to detect the steady state in the multivariate process.

Although this method performs well on some kinds of signals, it is also not robust in that one set of detection parameters is not able to handle signals of different characteristics, e.g., changing rate, noise level. Wu et al. [33-35] developed a robust method through Bayesian piecewise linear modeling and online inference of the latest line segment for steady state detection. Their method is much more accurate and robust on various types of signals.

\subsubsection{Variance Ratio Test}

All the aforementioned methods are for univariate systems. However, almost all systems are multivariate inherently. To guarantee that the system is steady, all the signals of the system have to be steady. Therefore, steady state detection of multivariate signals are desirable. To our best knowledge, there is a very limited number of multivariate steady state detection algorithms in the existing literature. Brown and Rhinehart.R [17] utilized the Cao's method [9] to detect the steady state of each signal separately. Once all the signals reach the steady state, the system is claimed steady. Mathematically, the overall test statistic can be written as $S S_{\text {process }}=\prod_{i=1}^{N} S S_{i}$ where $S S_{i}$ is the steady state status (0 denotes nonstationary while 1 denotes steady state) of the $i$-th signal. Once $S S_{\text {process }}=1$, the system or process is steady.

The Variance Ratio Test (VRT) [32] can be described as follows. In order to make the method more robust, some people propose to use exponentially weighted moving average (EWMA) method. The filter method applies three first-order filters to reduce the burden of computing to estimate deviation or variance from the measured trend. It evaluates the trend filtered based on the following equations. The first filter approximates the average value of the sample data. 


$$
X_{f, i}=\lambda_{1} X_{i}+\left(1-\lambda_{1}\right) X_{f, i-1}
$$

Where $X$ is the process variable. $X_{f}$ is the $X^{\prime}$ 's filtered value and $X_{f, i-1}$ is the previous filtered value of $X . \lambda_{1}$ is the filter factor $\left(0<\lambda_{1} \leq 1\right)$, and $i$ is the index of time sampling.

The recent observations are put a weight by a small $\lambda$, and this $\lambda$ can smooth more noises. The detection is however delayed due to the too low $\lambda$ when the process is at the steady state.

The second filter approach estimates the moving variance, which is exponentially weighted, based on the square of the difference between the filtered value and the measured value, which is estimated and given by Eq. (2.5).

$$
V_{f, i}^{2}=\lambda_{2}\left(X_{i}-X_{f, i-1}\right)^{2}+\left(1-\lambda_{2}\right) V_{f, i-1}^{2}
$$

Where $V_{f, i}^{2}$ is the filtered value of variance that is a measure value, and $V_{f, i-1}^{2}$ is the previous filtered value of variance based on variation from filtered trend.

Eq. (2.6) is a measurement of the variance applied in or ratio statistic or the numerator. Instead of the most newly updated value, the previous filtered value measure is applied to stop autocorrelation from biasing the variance estimate. $V_{f, i}^{2}$ is used to get the ratio equation simple in Eq. (2.6).

Based on the difference between successive data, the third filter method for evaluating the variance is to estimate the exponentially weighted moving variance. It is similar to the approach used to evaluate Eq. (2.6).

$$
\delta_{f, i}^{2}=\lambda_{3}\left(X_{i}-X_{i-1}\right)^{2}+\left(1-\lambda_{3}\right) \delta_{f, i-1}^{2}
$$

Where $\delta_{f, i}^{2}$ is the filtered value of variance's measure based on variation between sequential data and $\delta_{f, i-1}^{2}$ is the previous filtered value of variance. 
Eq. (2.7) provides estimation of denominator, while Eq. (2.6) provides the R-statistic calculation's the numerator. In order to prevent auto-correlation from biasing the variance estimate, the previous filtered value is applied to evaluate the ratio denominator and numerator and the recently updated variance will be replaced in both cases.

The variances ratio now may be estimated by Eq. (2.7) and Eq. (2.6). The R-test value is given by:

$$
\mathrm{R}=\frac{\left(2-\lambda_{1}\right) V_{f, i}^{2}}{\delta_{f, i}^{2}}
$$

The calculated value should be compared with its critical values in order to make sure whether it is steady or not. The variance is not calculated by Eq. (2.6) and Eq. (2.7). These equations just calculate the measurement of variances. According to the Eq. (2.8), the coefficient of $2-\lambda_{1}$ is used to scale this ratio in order to denote the variance ratio. In these filter methods, the $\lambda$ values can be associated with the holding window's length. $\lambda$ values are recommended [7] in order to guarantee or faster a steady state detection or greater confidence.

In extending this technique for a multivariate system analysis, we claim that the process is not steady when any process variable is not steady. If all process variables are at steady state, the process may be at steady state. This can easily be calculated by a single statistic:

$$
S S_{\text {process }}=\prod_{i=1}^{N} S S_{i}
$$

Where $N$ is the total number of variables in the process and $i$ is the variable numbers. $S S_{i}$ is 1 when this variable is at steady state and 0 when the variable is nonstationary. $S S_{\text {process }}$ is 1 after all variables are at steady state and 0 when all variables are nonstationary 


\subsubsection{Wavelet Transform Detection}

Jiang et al. [12] proposed wavelet transform method and to combine multiple monitoring indices into one through the Dempster's balance rule of combination. Clearly, this combination approach is just a generalization of the approach by Brown and Rhinehart [17] and therefore faces the same multiple testing problems.

Changes in the measurements can be detected by using WT modulus. Let $\psi(x)$ be the first-order wavelet which is the first-order derivative of scaling function, $\psi(x)=d \varphi(x) / d x$, then the Discrete Wavelet Transform(DWT) of $f(t)$ at dyadic scale $2^{j}$ and position $\mathrm{t}$ is given by

$$
W_{j} f(t)=f * \psi_{j}(t)=f *\left(2^{j} \cdot \frac{d \varphi_{j}}{d t}\right)(t)=2^{j} \frac{d}{d t}\left(f * \varphi_{j}\right)(t)
$$

where * denotes convolution operation. This equation indicates that $W_{j} f(t)$ which referred to as the first-order wavelet transform is proportional to the first derivative of $f(t)$ smoothed by a function $\varphi_{j}(t)$. The wavelet applied in this paper is Mallat wavelet [18]. The corresponding $\varphi(t)$ is a cubic spline, and thus $\psi(t)$ is a quadratic spline. If a modulus maximum $\left|W_{j} f(t)\right|$ is detected, then there is a corresponding sharp change in $f * \varphi_{j}$. Based on the above Eq. (2.10), an abnormality, which is a spike with a supernormal peak value and a short duration, can be detected by a couple of WT modulus maxima with opposite sign and large amplitudes, and then the data over the abnormality duration can be processed with average algorithm. After the abnormalities are processed, the signals will be decomposed and denoised by the soft threshold and then the denoised signals are reconstructed. Above method is called the wavelet-based multiscale process trends extraction.

Finally, after the original data is processed by the wavelet-based multi-scale processing technique, the processed data is used to calculate the steady-state index. The Eq. (2.10) again is used to calculate the $W_{j} f(t)$ which measures the variation in $f * \varphi_{j}(t)$. $W_{j} f(t)>0$ indicates an increase in $f * \varphi_{j}(t)$, and $W_{j} f(t)<0$ indicates a decrease. Large values of $\left|W_{j} f(t)\right|$ 
indicate sharp change in $f * \varphi_{j}(t)$, and small values of $\left|W_{j} f(t)\right|$ means gradual changes in $f *$ $\varphi_{j}(t)$. When $W_{j} f(t)=0, f * \varphi_{j}(t)$ indicates steady state. However, the zero-crossing points of $W_{j} f(t)$ should be distinguished. Different with zero points, the zero-crossing points are caused by the inflexion points, peak-value or valley-value points instead of the steady duration points of $f * \varphi_{j}(t)$. If $W_{j} f\left(t_{z}\right)=0,\left.\frac{d}{d t}\left(W_{j} f(t)\right)\right|_{t=t_{z}} \neq 0, t_{z}$ is called the zero-crossing points. Zerocrossing points of $W_{j} f(t)$ can be defined by

$$
W W_{j} f(t)=W_{j} f * \psi_{j}(t) \approx 2^{2 j} \frac{d^{2}}{d t^{2}}\left(f * \varphi_{j}\right)(t)
$$

where $W W_{j} f(t)$ is called the second-order WT and is proportional to the second derivative of $f * \varphi_{j}$. If $\left|W_{j} f(t)\right|<\delta_{1}$ and $\left|W W_{j} f(t)\right|>\delta_{2}$, the $t$ is a zero-crossing point where $\delta_{1}, \delta_{2}$ are small value constants. Based on the above method, the steady-state index, $0 \leq \beta \leq 1$, is computed to detect the steady state. $\beta=0$ indicates the unsteady, and $\beta=1$ indicates the steady [12]. For a multivariate system, every signal is processed and the steady-state index of every signal is calculated based on the mentioned methods. Then, the steady-state index $B(t)$ of a multivariate system is computed from the individual index of the key variables. For example, there is a multivariate system with $\mathrm{N}$ critical variables. $B(t)$ can be calculated following the Dempster's ruler of combination:

$$
B(t)=\prod_{i=1}^{N}\left[\beta_{i}(t)\right]^{\omega_{i} / \Sigma \omega_{i}}
$$

where $\beta_{i}(t)$ refers to the individual index of the ith key variable, $\omega_{i}$ is the weight, indicating the contribution of the $i$ th critical variable. $\omega_{i}$ is 1.0 as default. Thus, $B(t)=0$ indicate the unsteady state. $B(t)=1$ indicate the steady state, and $0<B(t)<1$ indicate the state between steady state and unsteady state. The results of simulation are show in Figure. 4 . 


\subsubsection{Other Methods}

To keep the overall significance level, the individual significance level is reduced or corrected from the well-known Sidak inequality, Kelly et.al [19] proposed a moving windowbased method where a residual Student $t$ test using the estimated mean of the process signal without any drift and the estimated standard-deviation of the underlying white-noise driving force is used to test if the signal is steady. To handle multivariate signals, they also followed Brown and Rhinehart.R's way [17] and chose to correct the individual significance level. However, similar to applying multiple univariate control charts on a multivariate data in SPC literature, this method faces the famous multiple testing problem [9] with inflated type I error or false discovery rate.

The basic assumption about any single signal is to suppose that in the window it can be operating by a non-zero slope multiplied using its qualified time as followed:

$$
x_{t}=m t+\mu+a_{t}
$$

where $m t$ is key drift component, $\mu$ is the mean of steady process, namely, it is the mean with the zero slope of the time window. $a_{t}$ is the i.i.d random series of error or white-noise sequence with standard deviation $\sigma_{a}$ and zero mean.

By the first difference of $x_{t}$ it is likely to unbiasedly estimate the drift module's slope $m$, $m t$ is the arithmetic mean of $x_{t}-x_{t-1}$ with $x_{t}$ 's $n$ sampled values in the window which in the time are similarly spaced. Then, the intercept $\mu$ is got by subtracting $m t$ from $x_{t}$ based on the above equation $x_{t}-m t=\mu+a_{t}$.

$$
\mu=\frac{1}{n}\left(\sum_{t=1}^{n} x_{t}-m \sum_{t=1}^{n} t\right)
$$

Now the estimate of the mean $\mu$ and drift slope $m$ of $x_{t}$ is got. The standard-deviation of the white-noise can be got as: 


$$
\sigma_{a}=\sqrt{\frac{1}{n-2} \sum_{t=1}^{n}\left(x_{t}-m t-\mu\right)^{2}}
$$

At a particular significance level $\alpha$ and degrees of freedom $n$, the Student's t critical value as a threshold value can be calculated. All of the essential information is available to check whether the process is steady or stationary about $\mu$. If $\left|x_{t}-\mu\right| \leq t_{c r i t} \sigma_{a}$, then $y_{t}=1$ else $y_{t}=0$

The summation of $y_{t}$ divided by $n$ is a portion about the chance that the null hypothesis is false. For example, a fraction of $95 \%$ indicates that there is $5 \%$ probability that the signals are not at steady state. For multivariate system, the individual significance level $\alpha^{\prime}$ is corrected from the overall significance level $\alpha$ derived from the Sidak inequality as followed:

$$
\alpha^{\prime}=1-(1-\alpha)^{1 / k}
$$

where $k$ is the number of key process variables. When the fraction of individual signal at the same time is beyond the overall confidence interval $(1-\alpha)$ over the same window, the multivariate system is steady or stationary.

In the batch processes, several methods are developed [15]. In these methods, various dimension reduction and feature extraction techniques are used first, such as multi-way principal component analysis (MPCA), dynamic principal component analysis (DPCA), and then one of the above-mentioned multivariate methods is used for steady state detection. Therefore, these methods are basically the same. The only difference lies in the preprocessing step, i.e., dimension reduction or feature extraction. Yao et.al [15] proposed Mahalanobis Distance Test (MDT) to reduce the dimension and extract the specific information from the signals.

The MDT method which is based on Mahalanobis Distance between the following batches and the first batch can be computed as follows: 


$$
\varphi_{i}=\sqrt{\left(\bar{X}_{1}-\bar{X}_{i}\right)^{T} \sum_{1}^{+}\left(\bar{X}_{1}-\bar{X}_{i}\right)}
$$

where $\varphi_{i}$ is the Mahalanobis distance. $X$ is a four dimensions data, $\sum_{1}^{+}$is the pseudoinverse of covariance matrix of $X_{1}$ which is regarded as the reference data. Then the two kinds of variance estimations are calculated using moving windows. One is ordinary variance estimation, another is a variance estimation of the mean square successive difference. The formulas of two kinds of variance estimations are defined as following:

$$
\begin{gathered}
S^{2}=\frac{\sum_{i=1}^{n}\left(\varphi_{i}-\bar{\varphi}\right)^{2}}{n-1} \\
\delta^{2}=\frac{\sum_{i=1}^{n-1}\left(\varphi_{i+1}-\varphi_{i}\right)^{2}}{n-1}
\end{gathered}
$$

Finally, the ratio of the mean square successive difference to the variance can be computed as:

$$
R=\frac{2 S^{2}}{\delta^{2}}
$$

This $R$ is suitable as a basis to judge whether this process is at steady state in MDT method. 


\section{Chapter 3: A EWMA Based LRT for Multivariate Steady State Detection}

\subsection{Introduction}

The efficient method is proposed to detect the steady state for the multivariate systems. This method is called Exponentially Weighted Moving Average-Likelihood Ratio Test (EWMALRT). This method estimates the covariance matrices using two different approaches, namely, the mean-squared-deviation and mean-squared-successive-difference. To avoid the usage of a moving window, the process means and the two covariance matrices are calculated recursively through exponentially weighted moving average. A likelihood ratio test is developed to compare the difference of the two covariance matrices and to detect the steady state.

\subsection{Methodology}

The computation of the covariance matrices is an multivariate extension of Cao [9]. Similarly, three EWMA filters are used to approximate the sample average and two covariance matrices.

The conventional sample covariance for a moving data window can be calculated as

$$
\widehat{\Sigma}_{x}=\frac{1}{N-1} \sum_{t=1}^{N}\left(X_{t}-\bar{X}_{t}\right)\left(X_{t}-\bar{X}_{t}\right)^{T}
$$

where $N$ is total number of observations in the moving window or the window size, $X_{t}$

is $t$-th the p-dimensional observation, and $\bar{X}_{t}$ is the mean of the $N$ observations. It is assumed that the observations are independently distributed as Gaussian distributions. To avoid the usage of moving window, the mean and the covariance can be calculated recursively through EWMA, which is computationally faster and requires much less storage. The moving average can be calculated as

$$
X_{f, t}=\lambda_{1} X_{t}+\left(1-\lambda_{1}\right) X_{f, t-1}
$$


where $0<\lambda_{1} \leq 1$ is the filtering coefficient. The covariance of $X_{f, t}$ can be derived as

$$
\Sigma_{X_{f, t}}=\left[\frac{\lambda_{1}\left(1-\left(1-\lambda_{1}\right)^{2 t}\right)}{2-\lambda_{1}}+\left(1-\lambda_{1}\right)^{2(t-1)}\right] \Sigma_{x}
$$

where $\Sigma_{x}$ is the true covariance matrix, which is assumed to be unchanged in the whole process. The detailed derivation of Eq. (3.3) is given in Appendix A. As time $t$ goes infinite,

$$
\Sigma_{X_{f, t}}=\frac{\lambda_{1}}{2-\lambda_{1}} \sum_{x}
$$

To calculate the covariance matrix recursively, the filtered value $X_{f, t-1}$ is used to replace the sample mean in Eq. (3.4), and the EWMA is also applied:

$$
\widehat{\Sigma}_{f, t}=\lambda_{2}\left(X_{t}-X_{f, t-1}\right)\left(X_{t}-X_{f, t-1}\right)^{T}+\left(1-\lambda_{2}\right) \widehat{\Sigma}_{f, t-1}
$$

where $0<\lambda_{2} \leq 1$ is the filtering coefficient. Alternatively, the mean squared successive difference can be used to estimate the covariance. Define $\sum_{s, t}$ as the expectation of the squared successive difference. It can be expressed as

$$
\Sigma_{s, t}=E\left(\left(X_{t}-X_{t-1}\right)\left(X_{t}-X_{t-1}\right)^{T}\right)
$$

Similarly, to calculate it recursively, another EWMA is applied as

$$
\widehat{\Sigma}_{s, t}=\lambda_{3}\left(X_{t}-X_{t-1}\right)\left(X_{t}-X_{t-1}\right)^{T}+\left(1-\lambda_{3}\right) \widehat{\Sigma}_{s, t-1}
$$

where $0<\lambda_{3} \leq 1$ is the filtering coefficient. 
In the stationary period, as $t$ approaches infinite, the expectations $E\left(\widehat{\Sigma}_{f, t}\right)$ and $E\left(\widehat{\Sigma}_{s, t}\right)$ can be proved to satisfy the following equations

and

$$
E\left(\widehat{\Sigma}_{f, t}\right)=\frac{2}{2-\lambda_{1}} \Sigma_{x}
$$

$$
E\left(\hat{\Sigma}_{s, t}\right)=2 \Sigma_{x}
$$

The proof of Eq.(3.8) and Eq. (3.9) are given in Appendix B and C, respectively.

Based on Eq.(3.8) and Eq.(3.9), the covariance matrix $\Sigma_{x}$ can be unbiasedly approximated using the following two approaches

$$
\hat{\Sigma}_{x}^{(1)}=\frac{2-\lambda_{1}}{2} \widehat{\Sigma}_{f, t}
$$

and

$$
\widehat{\Sigma}_{x}^{(2)}=\frac{1}{2} \widehat{\Sigma}_{s, t}
$$

In the steady state period, the covariance matrices estimated using Eq. (3.10)and Eq. (3.11) would be comparable. However, in the non-stationary period, the second approach using the squared successive differences would result in much larger dispersion than the first approach. To evaluate the difference, we use the likelihood ratio test (LRT), which is similar to LRT based control charts in statistical process control charts. The following absolute value of the likelihood ratio is utilized:

$$
\begin{gathered}
R_{t}=\left|\log \left(\frac{L\left(X_{t} \mid \mu=X_{f, t}, \Sigma=\hat{\Sigma}_{x}^{(1)}\right)}{L\left(X_{t} \mid \mu=X_{f, t}, \Sigma=\hat{\Sigma}_{x}^{(2)}\right)}\right)\right| \\
=\left|\frac{1}{2} \log \left(\frac{\left|\hat{\Sigma}_{x}^{(1)}\right|}{\left|\hat{\Sigma}_{x}^{(2)}\right|}\right)+\frac{1}{2}\left(X_{t}-X_{f, t}\right)^{T}\left(\hat{\Sigma}_{x}^{(1)}\right)^{-1}\left(X_{t}-X_{f, t}\right)-\frac{1}{2}\left(X_{t}-X_{f, t}\right)^{T}\left(\hat{\Sigma}_{x}^{(2)}\right)^{-1}\left(X_{t}-X_{f, t}\right)\right|
\end{gathered}
$$


In order to make the $R_{\mathrm{t}}$ calculated by the likelihood ratio test more stable and sensitive to the detection of small drift, the EWMA is also used as follows

$$
D_{t}=\lambda_{4} R_{t}+\left(1-\lambda_{4}\right) D_{t-1}
$$

In the non-stationary period, the index $D_{t}$ is expected to be larger than 0 while in the stationary period, it will approach to 0 . We denote this method as EWMA-LRT. In the following section, we use numerical studies and real case study to demonstrate the effectiveness of the proposed approach.

\subsection{Illustration of the EWMA-LRT Method}

In this section we use simulated signals is to illustrate the detection process of the EWMALRT algorithm for multivariate system. The simulated signals are generated by bias functions and noise. These bias functions are composed of an initial transient state and a steady-state. There are four bias functions used in this article: linear function, quadratic function, exponential function and oscillating function, as show in Table 3.1. These bias functions have been widely used in evaluating the initial bias truncation algorithms for discrete-event simulations [20].

To illustrate how the algorithm works, four-dimensional $(p=4)$ signals are simulated with each bias function simulating one dimension. For these four functions, $h=1$ (the height of the signal), $T=600$ (the total number of observations of the signal). Two steady state times are considered, $T_{0}=200$ and $T_{0}=300$, which represents two different initial bias changing rates. For the oscillating function, $f=30$ (the total of 10 peaks and troughs). The signal noise is set to $\sum=0.01 I_{4}$, where $I_{4}$ is a $4 \times 4$ identity matrix. The filter factors for the EWMA-LRT method are selected as: $\lambda_{1}=0.1, \lambda_{2}=0.1, \lambda_{3}=0.1, \lambda_{4}=0.1$. The threshold $D_{c}$ is set to 0.5 .

Figure 3.1 shows that the steady-state detection process for the two randomly generated four-dimensional output. Clearly, the detection index $D_{t}$ rapidly increases at the beginning, and then gradually decreases until the signal enters into steady state, where $D_{t}$ is stable around a value 
close to 0 . Once the value of $D_{t}$ is below the threshold 0.5 , the multivariate signal is claimed steady. The detection times are $T^{*}=205$ and $T^{*}=288$, respectively, which are very close to the true values.

\section{Table 3.1: Four Mean Bias Functions and Their Shapes}

\begin{tabular}{|c|c|c|}
\hline Bias Type & Function Form & Shape \\
\hline Linear & $y(t)=\left\{\begin{array}{l}\frac{t}{T_{0}} h, \quad t=1,2 \ldots, T_{0} \\
h, \quad t=T_{0}+1, \ldots, n\end{array}\right.$ & \\
\hline Quadratic & $y(t)=\left\{\begin{array}{c}h\left[1-\frac{\left(t-T_{0}\right)^{2}}{\left(T_{0}-1\right)^{2}}\right], \quad t=1,2 \ldots, T_{0} \\
h, \quad t=T_{0}+1, \ldots, n\end{array}\right.$ & \\
\hline Exponential & $y(t)=\left\{\begin{array}{c}h\left[1-10^{\left.\frac{1-t}{T_{0}-1}\right], \quad t=1,2 \ldots ., T_{0}}\right. \\
y\left(T_{0}\right), \quad t=T_{0}+1, \ldots, n\end{array}\right.$ & \\
\hline Oscillating & $y(t)=\left\{\begin{array}{c}h \frac{T_{0}-t}{T_{0}-1} \sin \left(\frac{\pi t}{f}\right), \quad t=1,2 \ldots ., T_{0} \\
0, \quad t=T_{0}+1, \ldots, n\end{array}\right.$ & \\
\hline
\end{tabular}

\subsection{Performance Evaluation and Comparison}

In this section, we compare the proposed method with several existing algorithms. The performance measures for steady state detection often include the closeness of detected time to the actual one, and the false alarm rate (FAR) [21]. The closeness measure alone is often inadequate for detection performance evaluation since it does not consider the false alarm (early detection) or detection delay. For example, for the same detection closeness, the detection delay is often better 
than early detection in many practical applications. To consider both detection closeness and FAR, we use another evaluation metric, the weighed standard error (WSE) [21, 22]:

(a)
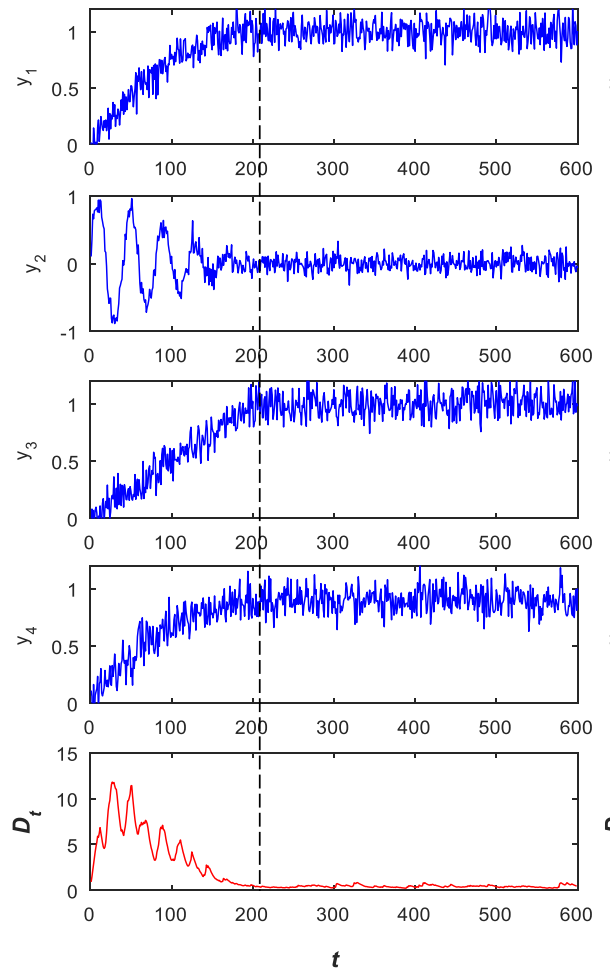

(b)
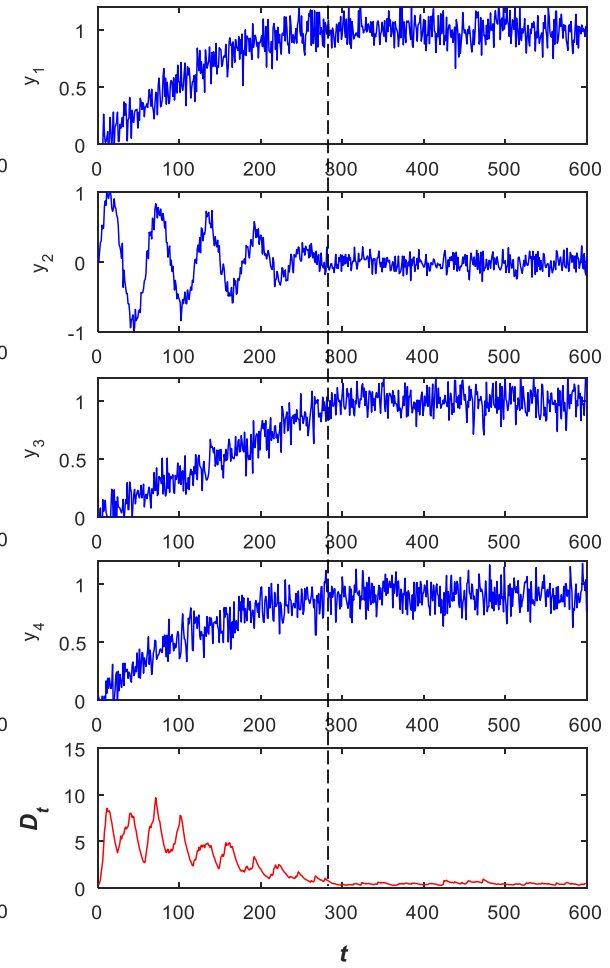

Figure 3.1: Illustration of the steady state detection of EWMA-LRT: (a) $T_{0}=200$, the detected steady state time $T^{*}=205$, (b) $T_{0}=300$, the detected steady-state time $T^{*}=288$. The vertical dashed line indicates the detected steady state time.

$$
\mathrm{WSE}=\sqrt{\frac{1}{N} \sum_{i=1}^{N} w\left(T_{i}^{*}\right)\left(T_{i}^{*}-T_{0}\right)^{2}}
$$

where $N$ is the total number of replications, $T_{i}^{*}$ and $T_{0}$ are the detected value and true value respectively for the $i$ th multivariate output, and $w\left(T_{i}^{*}\right)$ is the penalty weight expressed as 


$$
w\left(T_{i}^{*}\right)=\left\{\begin{array}{lr}
w \in(0,1], & \text { if } T_{i}^{*} \geq T_{0} \\
1, & \text { if } T_{i}^{*}<T_{0}
\end{array}\right.
$$

If $w\left(T_{i}^{*}\right) \equiv 1$, then WSE is just the closeness measure. If $w<1$, then a lower penalty is put on detection delay than early detection. $w$ can be treated as the penalty ratio of the detection delay to the early detection. To show how many signals are early-detected, we also use the FAR as an auxiliary metric.

Table 3.2: Noise Types and Their Parameters

\begin{tabular}{ccc}
\hline \hline Type & Equation & Parameter \\
\hline $\operatorname{AR}(0)$ & $\psi_{t}=\epsilon_{t}$ & $\epsilon_{t} \sim N\left(0, \sigma^{2} I_{p}\right)$ \\
$\operatorname{AR}(1)$ & $\psi_{t}=\phi_{1} \psi_{t-1}+\epsilon_{t}$ & $\epsilon_{t} \sim N\left(0, \sigma^{2} I_{p}\right), \phi_{1}=0.4$ \\
$\operatorname{AR}(2)$ & $\psi_{t}=\phi_{2} \psi_{t-1}+\phi_{3} \psi_{t-2}+\epsilon_{t}$ & $\epsilon_{t} \sim N\left(0, \sigma^{2} I_{p}\right), \phi_{2}=-0.25, \phi_{3}=0.5$ \\
\hline
\end{tabular}

In the performance evaluation and comparison, $p=4$ and 8 are selected and each dimension is simulated using a bias function randomly selected from the four given in Table 3.1. The height of the signal is set to $h=1$ and $h=2$. To simulate different bias severity, two values for $T_{0}$ are selected: $T_{0}=200$ and $T_{0}=300$. It is assumed that all dimensions reach steady state at the same time. The length of the signal is set to be 600.100 replications are generated for each set of signal parameters. Three types of Gaussian noise are used to test the algorithm: (1) no autocorrelation, denoted by $\mathrm{AR}(0)$; (2) first-order autoregressive correlation, denoted by $\mathrm{AR}(1)$; (3) second-order autoregressive correlation, denoted by $\operatorname{AR}(2)$. The noises types and their parameters are listed in Table 3.2. Different noise amplitudes are also considered: for $\operatorname{AR}(0), \sigma=$ $0.06,0.1,0.14$; for $\operatorname{AR}(1)$ and $\operatorname{AR}(2), \sigma=0.055,0.089,0.13$ and $\sigma=0.045,0.071,0.106$ respectively to match the noise variances of $\mathrm{AR}(0)$.

The proposed EWMA-LRT method is compared with the well-known Variance Ratio Test (VRT) [9], SSD method to detect non-stationary drifts [19] and Wavelet Transform Detection (WTD) method [12]. Since VRT is a univariate method, it is applied to each dimension of the multivariate signal and then the largest detection value is selected as the steady state time. It means that a multivariate process is not steady if any process variable is at transient state, while it is steady 
once all process variables enter into steady state. For all methods, the optimal filter factors, threshold values and other parameters are selected by minimizing the overall WSE. For the SSD method, the optimal window size of 22 is selected. The overall significance level $\alpha$ is set as 0.05 . Based on the Sidak inequality, the individual significance level should be 0.0127 .For WTD method, the process trends are extracted from the raw measurements to eliminate the random noise and abnormalities. Then, the steady-state index is calculated for every process variable and the second-order WT modulus is used to address the zero-crossing points. Finally, the steady-state index of a multivariate system is computed from the Dempster's ruler.
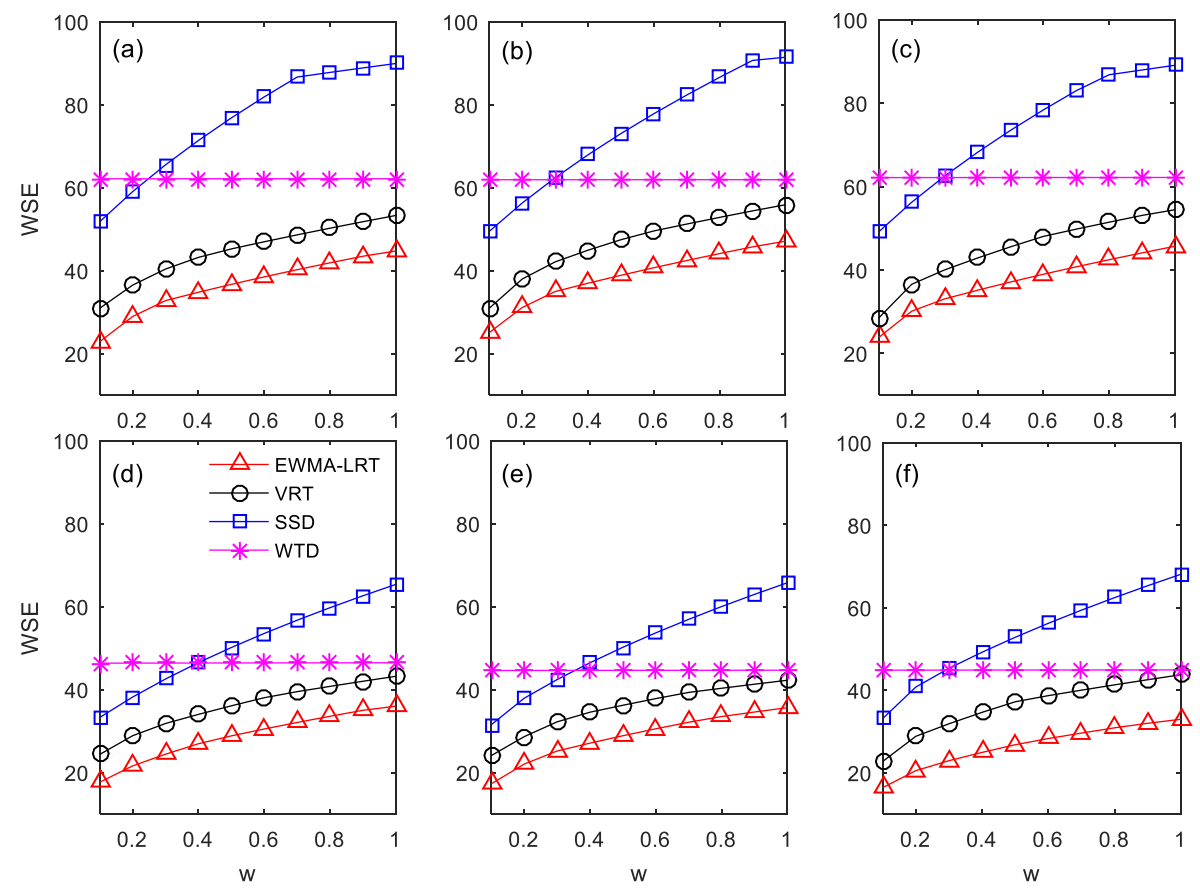

Figure 3.2: The WSE of EWMA-LRT, VRT, SSD and WTD method as a function of penalty weight $w$ : top row $(\mathrm{a}, \mathrm{b}, \mathrm{c}): p=4$; bottom row $(\mathrm{d}, \mathrm{e}, \mathrm{f}): p=8 ;(\mathrm{a}, \mathrm{d}): \operatorname{AR}(0),(\mathrm{b}, \mathrm{e}): \operatorname{AR}(1)$ and $(\mathrm{c}$, f): $\operatorname{AR}(2)$.

Figure 3.2 shows the WSE as a function of $w$ for EWMA-LRT, VRT, SSD and WTD under different $\mathrm{p}$ and noise types. Table 3.3 and Table 3.4 shows the detailed detection results for $\mathrm{w} \equiv 1$, i.e., closeness and FAR, for $p=4$. For space limitation, the detailed detection results for $p=8$ 
is not provided here. The proposed EWMA-LRT outperforms other methods significantly for all cases in terms of WSE. For WTD, the WSE is almost unchanged when w varies. The reason is that WTD has a very high FAR, e.g., almost 90\% for all cases; besides, increasing $T_{0}$ or noise $\sigma$ will reduce the detection closeness and increase the FAR for all methods, as shown in Table 3.3 and Table 3.4. The reason is that more initial bias will be immersed into signal noise and becomes undetectable when the noise amplitude is increased or the mean shifting rate before steady state is reduced; in addition, the performances are almost the same under different noise types for all methods, indicating that they are also applicable for autoregressive noises with mild or medium autocorrelation. Increasing the dimension from $p=4$ to $p=8$, the performances of all methods are improved, which is an important advantage of multivariate detection method over univariate method.

\subsection{Conclusion}

(1) An efficient method named Exponentially Weighted Moving Average-Likelihood Ratio Test (EWMA-LRT) has been developed to detect the steady state of the multivariate systems.

(2) The covariance matrices are estimated through two approaches, the mean-squared-deviation and the mean-squared successive difference. Two covariance matrices are computed recursively through EWMA. While in steady state period, both these two covariance matrices are unbiased estimator of the true covariance matrix. Based on these findings, a likelihood ratio test is proposed to compare these two estimated covariance matrices.

(3) The performance of the proposed method was evaluated and compared with existing ones through numerical studies. The simulation results demonstrated that the proposed methods outperforms others for various types of signals.

(4) The results of this research provide useful guidelines for establishing an online steady state detection scheme. It should be pointed that the detection parameters, including the filtering weights and detection threshold, are essential to achieve a reliable detection. 
Table 3.3: Detailed Comparison of EWMA-LRT, VRT, SSD and WTD for the Case of $p=4, w=1$

\begin{tabular}{|c|c|c|c|c|c|c|c|c|c|c|c|}
\hline \multicolumn{4}{|c|}{$\begin{array}{l}\text { Signal } \\
\end{array}$} & \multicolumn{4}{|c|}{$\mathrm{WSE}(w \equiv 1)$} & \multicolumn{4}{|c|}{ FAR } \\
\hline Noise & $T_{0}$ & $h$ & $\sigma$ & LRT & VRT & SSD & WTD & LRT & VRT & SSD & WTD \\
\hline \multirow{12}{*}{$\mathrm{AR}(0)$} & \multirow{6}{*}{200} & \multirow{3}{*}{1} & 0.06 & 31.8 & 47.5 & 57.2 & 32.9 & 0.04 & 0.01 & 0.2 & 0.99 \\
\hline & & & 0.10 & 24.2 & 30.9 & 73.5 & 38.7 & 0.28 & 0.15 & 0.59 & 0.98 \\
\hline & & & 0.14 & 38.1 & 41.4 & 83.3 & 48.6 & 0.62 & 0.59 & 0.71 & 0.95 \\
\hline & & \multirow{3}{*}{2} & 0.06 & 45.9 & 67.1 & 65.8 & 31.8 & 0 & 0 & 0.02 & 1 \\
\hline & & & 0.10 & 36.8 & 52.2 & 60.2 & 30.7 & 0.01 & 0 & 0.15 & 0.98 \\
\hline & & & 0.14 & 30.4 & 43.0 & 59.2 & 33.0 & 0.05 & 0.01 & 0.34 & 0.99 \\
\hline & \multirow{6}{*}{300} & \multirow{3}{*}{1} & 0.06 & 26.4 & 39.2 & 74.1 & 67.3 & 0.24 & 0.1 & 0.5 & 0.98 \\
\hline & & & 0.10 & 56.5 & 65.3 & 118.3 & 92.3 & 0.71 & 0.6 & 0.78 & 0.95 \\
\hline & & & 0.14 & 94.6 & 90.5 & 166.6 & 120.9 & 0.97 & 0.81 & 0.93 & 0.99 \\
\hline & & \multirow{3}{*}{2} & 0.06 & 39.0 & 54.2 & 61.8 & 54.3 & 0.01 & 0.01 & 0.17 & 1 \\
\hline & & & 0.10 & 31.5 & 40.5 & 75.6 & 53.9 & 0.05 & 0.08 & 0.4 & 0.98 \\
\hline & & & 0.14 & 35.2 & 39.3 & 115.1 & 69.9 & 0.41 & 0.41 & 0.73 & 0.99 \\
\hline \multirow{12}{*}{$\mathrm{AR}(1)$} & \multirow{6}{*}{200} & \multirow{3}{*}{1} & 0.06 & 35.7 & 36.2 & 45.1 & 32.8 & 0.02 & 0.02 & 0.43 & 0.97 \\
\hline & & & 0.10 & 27.4 & 26.3 & 57.6 & 37.1 & 0.16 & 0.41 & 0.58 & 0.95 \\
\hline & & & 0.14 & 40.3 & 54.2 & 88.5 & 38.8 & 0.64 & 0.82 & 0.8 & 0.93 \\
\hline & & \multirow{3}{*}{2} & 0.06 & 51.2 & 53.5 & 50.6 & 37.0 & 0 & 0 & 0.11 & 1 \\
\hline & & & 0.10 & 38.6 & 45.4 & 39.5 & 35.9 & 0.01 & 0.01 & 0.29 & 1 \\
\hline & & & 0.14 & 30.3 & 30.8 & 47.2 & 30.9 & 0.02 & 0.12 & 0.42 & 0.99 \\
\hline & \multirow{6}{*}{300} & \multirow{3}{*}{1} & 0.06 & 25.6 & 36.5 & 108.1 & 64.6 & 0.21 & 0.42 & 0.79 & 0.99 \\
\hline & & & 0.10 & 45.7 & 78.6 & 130.9 & 85.8 & 0.63 & 0.77 & 0.81 & 0.98 \\
\hline & & & 0.14 & 107.4 & 115.8 & 160.7 & 118.7 & 0.96 & 0.91 & 0.95 & 0.99 \\
\hline & & \multirow{3}{*}{2} & 0.06 & 43.0 & 44.8 & 62.1 & 61.0 & 0.01 & 0.02 & 0.31 & 1 \\
\hline & & & 0.10 & 29.6 & 34.3 & 95.2 & 70.3 & 0.12 & 0.24 & 0.63 & 0.99 \\
\hline & & & 0.14 & 30.7 & 50.6 & 115.3 & 64.5 & 0.3 & 0.65 & 0.79 & 0.97 \\
\hline \multirow{12}{*}{$\mathrm{AR}(2)$} & \multirow{6}{*}{200} & \multirow{3}{*}{1} & 0.06 & 42.9 & 46.0 & 51.3 & 37.9 & 0.01 & 0.01 & 0.22 & 0.97 \\
\hline & & & 0.10 & 35.3 & 30.1 & 68.6 & 42.5 & 0.11 & 0.27 & 0.61 & 0.94 \\
\hline & & & 0.14 & 28.5 & 44.4 & 87.1 & 40.9 & 0.39 & 0.75 & 0.73 & 0.95 \\
\hline & & \multirow{3}{*}{2} & 0.06 & 60.2 & 60.7 & 71.8 & 31.9 & 0 & 0 & 0.02 & 1 \\
\hline & & & 0.10 & 47.8 & 47.9 & 57.6 & 35.3 & 0 & 0 & 0.14 & 1 \\
\hline & & & 0.14 & 41.7 & 38.4 & 63.2 & 32.0 & 0 & 0.04 & 0.34 & 0.95 \\
\hline & \multirow{6}{*}{300} & \multirow{3}{*}{1} & 0.06 & 33.3 & 36.3 & 97.8 & 66.7 & 0.08 & 0.16 & 0.59 & 0.98 \\
\hline & & & 0.10 & 43.8 & 74.5 & 124.1 & 95.0 & 0.56 & 0.74 & 0.83 & 0.97 \\
\hline & & & 0.14 & 74.7 & 106.5 & 161.2 & 108.5 & 0.79 & 0.83 & 0.86 & 0.97 \\
\hline & & \multirow{3}{*}{2} & 0.06 & 50.1 & 50.6 & 62.9 & 64.3 & 0 & 0.02 & 0.18 & 0.97 \\
\hline & & & 0.10 & 36.2 & 36.8 & 75.2 & 66.2 & 0.05 & 0.18 & 0.46 & 0.96 \\
\hline & & & 0.14 & 32.9 & 32.0 & 85.5 & 66.8 & 0.18 & 0.29 & 0.62 & 0.98 \\
\hline & Overa & & & 45.8 & 54.6 & 90.2 & 62.1 & 0.24 & 0.29 & 0.50 & 0.98 \\
\hline
\end{tabular}


Table 3.4: Detailed Comparison of EWMA-LRT, VRT, SSD and WTD for the Case of $p=8, w=1$

\begin{tabular}{|c|c|c|c|c|c|c|c|c|c|c|c|}
\hline \multicolumn{4}{|c|}{ Signal } & \multicolumn{4}{|c|}{$\mathrm{WSE}(w \equiv 1)$} & \multicolumn{4}{|c|}{ 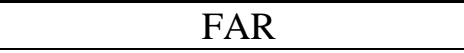 } \\
\hline Noise & $T_{0}$ & $h$ & $\sigma$ & LRT & VRT & SSD & WTD & LRT & VRT & SSD & WTD \\
\hline \multirow{12}{*}{$\mathrm{AR}(0)$} & \multirow{6}{*}{200} & \multirow{3}{*}{1} & 0.06 & 29.0 & 40.5 & 55.9 & 13.6 & 0.01 & 0 & 0.08 & 0.6 \\
\hline & & & 0.10 & 16.2 & 17.6 & 62.0 & 23.7 & 0.24 & 0.28 & 0.21 & 0.59 \\
\hline & & & 0.14 & 17.6 & 19.4 & 68.0 & 35.4 & 0.7 & 0.72 & 0.48 & 0.72 \\
\hline & & \multirow{3}{*}{2} & 0.06 & 49.1 & 57.8 & 61.3 & 14.9 & 0 & 0 & 0 & 0.81 \\
\hline & & & 0.10 & 33.4 & 47.0 & 62.4 & 17.5 & 0 & 0 & 0.01 & 0.59 \\
\hline & & & 0.14 & 23.8 & 32.7 & 56.3 & 16.3 & 0.03 & 0 & 0.08 & 0.6 \\
\hline & \multirow{6}{*}{300} & \multirow{3}{*}{1} & 0.06 & 18.7 & 20.5 & 55.6 & 41.2 & 0.18 & 0.22 & 0.1 & 0.79 \\
\hline & & & 0.10 & 32.0 & 40.6 & 56.7 & 72.8 & 0.84 & 0.69 & 0.36 & 0.89 \\
\hline & & & 0.14 & 79.9 & 86.0 & 79.1 & 107.1 & 0.98 & 0.93 & 0.66 & 0.92 \\
\hline & & \multirow{3}{*}{2} & 0.06 & 37.4 & 48.9 & 80.9 & 24.3 & 0 & 0 & 0 & 0.82 \\
\hline & & & 0.10 & 23.0 & 30.7 & 71.7 & 33.1 & 0.04 & 0.08 & 0.05 & 0.79 \\
\hline & & & 0.14 & 19.1 & 25.7 & 68.2 & 54.6 & 0.33 & 0.41 & 0.1 & 0.8 \\
\hline \multirow{12}{*}{$\operatorname{AR}(1)$} & \multirow{6}{*}{200} & \multirow{3}{*}{1} & 0.06 & 30.2 & 35.3 & 68.5 & 15.7 & 0.01 & 0 & 0.02 & 0.63 \\
\hline & & & 0.10 & 22.9 & 18.1 & 49.5 & 25.0 & 0.16 & 0.5 & 0.24 & 0.67 \\
\hline & & & 0.14 & 21.9 & 27.1 & 54.3 & 34.7 & 0.55 & 0.83 & 0.48 & 0.74 \\
\hline & & \multirow{3}{*}{2} & 0.06 & 45.3 & 53.3 & 71.2 & 15.9 & 0 & 0 & 0 & 0.79 \\
\hline & & & 0.10 & 36.0 & 40.8 & 57.8 & 16.2 & 0 & 0 & 0.02 & 0.7 \\
\hline & & & 0.14 & 30.2 & 30.1 & 59.3 & 16.4 & 0.02 & 0.04 & 0.03 & 0.61 \\
\hline & \multirow{6}{*}{300} & \multirow{3}{*}{1} & 0.06 & 22.5 & 23.6 & 67.3 & 39.3 & 0.09 & 0.43 & 0.05 & 0.93 \\
\hline & & & 0.10 & 29.5 & 53.8 & 59.7 & 70.3 & 0.65 & 0.85 & 0.35 & 0.91 \\
\hline & & & 0.14 & 71.4 & 84.5 & 76.8 & 103.1 & 0.94 & 0.98 & 0.62 & 0.98 \\
\hline & & \multirow{3}{*}{2} & 0.06 & 36.5 & 44.2 & 79.5 & 24.9 & 0.01 & 0.02 & 0 & 0.85 \\
\hline & & & 0.10 & 25.8 & 23.0 & 75.8 & 31.7 & 0.05 & 0.21 & 0.02 & 0.89 \\
\hline & & & 0.14 & 24.4 & 25.2 & 61.8 & 48.5 & 0.23 & 0.66 & 0.14 & 0.85 \\
\hline \multirow{13}{*}{$\operatorname{AR}(2)$} & \multirow{6}{*}{200} & \multirow{3}{*}{1} & 0.06 & 28.2 & 39.7 & 60.7 & 19.7 & 0.01 & 0 & 0.05 & 0.59 \\
\hline & & & 0.10 & 15.7 & 15.4 & 51.7 & 20.6 & 0.22 & 0.37 & 0.27 & 0.58 \\
\hline & & & 0.14 & 22.3 & 30.6 & 54.4 & 34.4 & 0.73 & 0.74 & 0.46 & 0.67 \\
\hline & & \multirow{3}{*}{2} & 0.06 & 48.5 & 55.3 & 70.3 & 18.8 & 0 & 0 & 0 & 0.79 \\
\hline & & & 0.10 & 33.0 & 49.2 & 64.4 & 18.7 & 0 & 0 & 0.02 & 0.66 \\
\hline & & & 0.14 & 25.0 & 37.2 & 65.5 & 17.3 & 0.03 & 0.02 & 0.05 & 0.64 \\
\hline & \multirow{6}{*}{300} & \multirow{3}{*}{1} & 0.06 & 19.2 & 29.4 & 75.2 & 35.1 & 0.08 & 0.25 & 0.04 & 0.81 \\
\hline & & & 0.10 & 35.6 & 58.1 & 58.9 & 76.2 & 0.85 & 0.83 & 0.36 & 0.89 \\
\hline & & & 0.14 & 61.0 & 70.6 & 82.7 & 96.7 & 0.98 & 0.95 & 0.6 & 0.96 \\
\hline & & \multirow{3}{*}{2} & 0.06 & 37.1 & 47.6 & 77.5 & 24.8 & 0 & 0 & 0.01 & 0.81 \\
\hline & & & 0.10 & 22.4 & 30.5 & 79.3 & 31.6 & 0.05 & 0.08 & 0.01 & 0.83 \\
\hline & & & 0.14 & 14.2 & 31.0 & 68.9 & 56.9 & 0.28 & 0.39 & 0.11 & 0.82 \\
\hline & Over & & & 36.0 & 43.2 & 65.4 & 46.5 & 0.26 & 0.32 & 0.17 & 0.76 \\
\hline
\end{tabular}




\section{Chapter 4: Case Study}

\subsection{Introduction}

In this chapter, the application to the Micro/nanoparticle dispersion process will be given to demonstrate the proposed method can detect the steady state for multivariate system, and the EWMA-LRT can be used in many areas. As we know, due to high surface-to-volume ratio and high surface energy, the micro/nanoparticles tend to agglomerate and cluster together, which may significantly limit their effectiveness. However, they must be dispersed each other before use. Ultrasonic cavitation is an effective method to disperse them. At the ultrasonic power level, the steady state of the Cavitation Noise Power (CNP) signals corresponds to the maximum extent of dispersion. CNP signals is the integration of cavitation noise spectrum over frequency in a logarithmic scale.

\subsection{Application to the Micro/nanoparticle Dispersion Process}

In this chapter, the proposed method is applied to cavitation noise signals obtained from the micro/nanoparticle dispersion process [11]. Recently, micro/nanoparticles have attracted significant scientific interests, due to a wide variety of potential applications in biomedical, optical, electronic, and mechanical fields. However, in actual applications, due to high surface-to-volume ratio and high surface energy, the micro/nanoparticles often agglomerate or cluster together, which may significantly limit their effectiveness. Therefore, the micro/nanoparticles must be dispersed before use. Ultrasonic cavitation is an effective method to disperse micro/nanoparticles. It is found that when the cavitation noise signals enter into steady state, the dispersion process is finished [11]. Thus, the dispersion process can be monitored by detecting the steady state of cavitation noise signals.

Ultrasonic cavitation is a good technology to disperse particles in the liquid [38]. Ultrasonic waves are high intensive so that they can generate important non-linear effects in the liquid and be dependable for handling fluid-based materials. The essential thought is to shoot a ultrasound beam via the particle-liquid framework. At that point due to nearby violent pressure variations produced 
by the ultrasonic vibrations, we are going have a "cavitation" phenomenon, which alludes to the foundation, growth, fluctuation, and implosive breakdown of vapor bubbles or gas triggered by the ultrasonic sound in the liquids [11], as shown in Figure 4.1.

The cavitation is classified in two types based on the duration of the bubbles: steady cavitation and nonstationary cavitation. The bubbles non-linearly oscillate around the size of the balance for the steady cavitation. They will last for lots of acoustic pressure cycles and are relatively stable. Meanwhile, the bubbles usually oscillate for a much shorter time during transient cavitation. They grow explosively into a hole with the size of their innovative sizes many times and then violently collapse. After these bubbles break, they will get transient micro "hot spot". In addition, acoustic streaming can lead to violent agitation in liquids. Because of these intense effects, the cavitation breaks particles clustering effectively into dispersion particles and can mix well in liquids. Ultrasound cavitation is therefore promising as a reliable and cost-effective tool for the dispersion of nanoparticles in metal melt is given Figure 4.1.

(a)
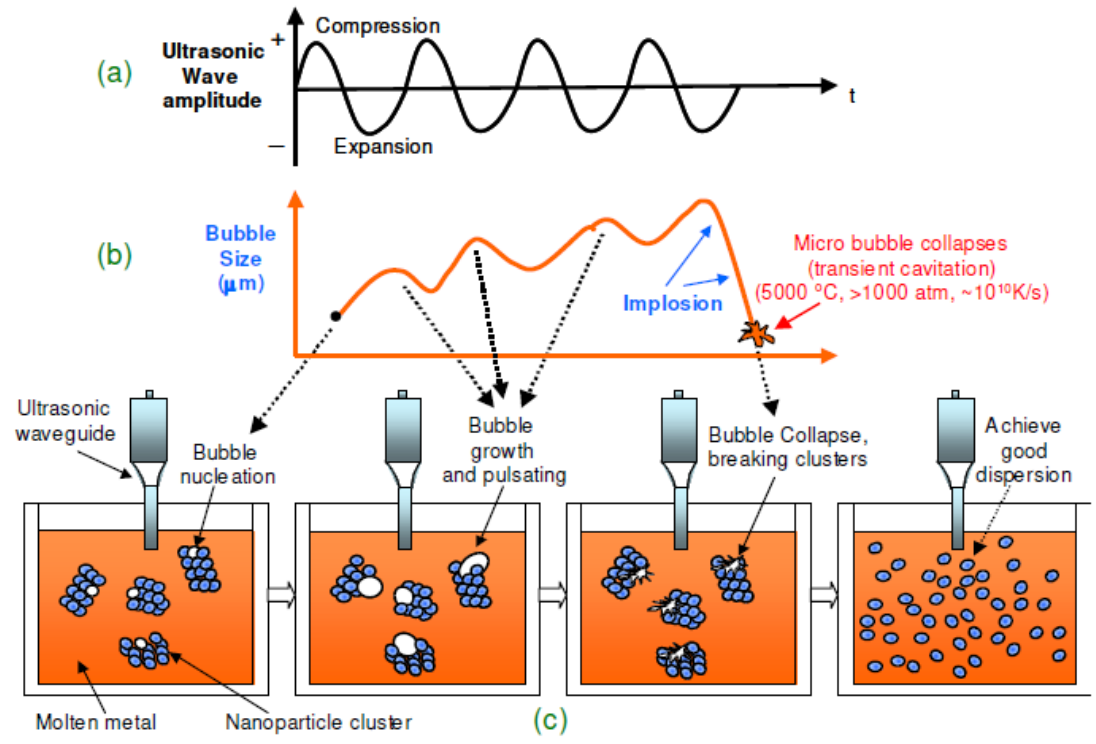

Figure 4.1: Ultrasonic dispersion of nanoparticles 
Until now there are a number of methods for cavitation detection and monitoring. The analysis of cavitation noise spectrum is the most popular method, because of its low cost, ease of implementation and ability to get many cavitation information by acoustic transducers. In this research we can use the cavitation noise spectrum to capture some information about dispersion process, such as cavitation noise power (CNP). CNP will be described by the two different indices. One is CNP-1, another is CNP-2. CNP-1 is defined as the integration of cavitation noise spectrum from $0-200 \mathrm{KHz}$ in a logarithmic scale to enhance the "white noise" contribution. In this dissertation, we mainly use the CNP-1 to detect the steady state.

$$
\mathrm{CNP} 1=\int A(f) d f \approx \sum A(f) d f
$$

Where,

$A(f)=$ Discrete Fourier Transform (DFT) amplitude in the logarithmic scale $f=$ Frequency

CNP-2 is defined as the averaged square of the cavitation noise signal in each second.

$$
\mathrm{CNP} 2=\frac{\sum_{i=1}^{n} U_{i}^{2}}{n}
$$

Where,

$U_{i}=$ Cavitation noise signal

$n=$ The number of samples in every second.

\subsection{Simulation Results}

To get the evolution of cavitation's noise, Wu et al. [11] developed a univariate monitoring index, termed as cavitation noise power (CNP), which is the integration of cavitation noise spectrum over frequency in a logarithmic scale. However, such information fusion will inevitably result in information loss. Here we consider spectrum amplitudes of four critical frequency components, $0 \mathrm{KHz}, 20 \mathrm{KHz}, 40 \mathrm{KHz}$ and $60 \mathrm{KHz}$, which are harmonics, subharmonics, and ultraharmonics. They are directly related with the physical dispersion process. 

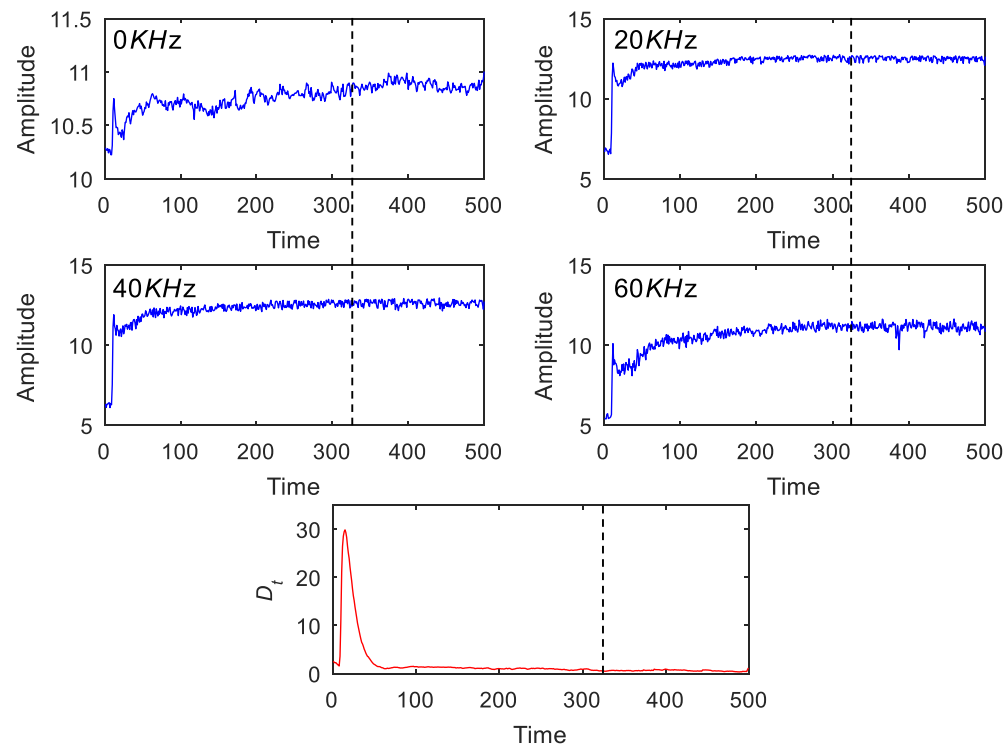

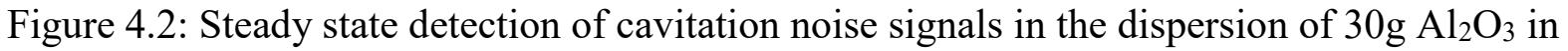
water with ultrasonic power $40 \mathrm{~W}$.

Figure 4.2 shows the steady state detection of the four-dimensional spectrum amplitude signals in the dispersion of $30 \mathrm{~g} \mathrm{Al}_{2} \mathrm{O}_{3}$ particles with ultrasonic power $40 \mathrm{~W}$. The same detection parameters $\left(\lambda_{i}=0.1, i=1, \ldots, 4, D_{c}=0.5\right)$ are used to detect and evaluate the steady state. The detected steady state time is 327, which is higher than using univariate CNP signal (around 300). It is expected since CNP signal has information loss. If the process reaches steady state, CNP signal will be steady. However, the reverse is not necessarily true, i.e., the steady state of CNP signal does not necessarily indicate a steady state of the whole system.

\subsection{Conclusion}

Ultrasonic cavitation is an effective method to disperse micro/nanoparticles. At the ultrasonic power level, the steady state of Cavitation Noise Power (CNP) signals corresponds to the maximum extent of dispersion. Thus, detecting the steady state of CNP signals can well monitor this dispersion process. The simulation result demonstrates that the proposed method can identify the steady state in the Micro/nanoparticle dispersion process. The detected steady state time is 327 that is very close to the true value (around 300). 


\section{Chapter 5: Conclusion and Recommendation for Future Work}

In this study, we have developed a new online method to detect the steady state of the multivariate systems. The covariance matrices are estimated through two approaches, the meansquared-deviation and the mean-squared successive difference. To reduce the computational cost and avoid the usage of moving windows, these two covariance matrices are computed recursively through exponentially weighted moving average. In the non-stationary period, the dispersion of the covariance matrix estimated using mean-squared successive difference is expected to be larger than that using mean-squared-deviation. While in steady state period, both these two covariance matrices are unbiased estimator of the true covariance matrix. Based on these findings, a likelihood ratio test is proposed to compare these two estimated covariance matrices.

The performance of the proposed method was evaluated and compared with existing ones through numerical studies. The simulation results demonstrated that the proposed methods outperforms others for various types of signals. A real case study is also performed to show its application and effectiveness. The results of this research provide useful guidelines for establishing an online steady state detection scheme. It should be pointed that the detection parameters, including the filtering weights and detection threshold, are essential to achieve a reliable detection. In practical applications, these parameters can be tuned using simulated signals with various types of bias functions and bias severities

My future work will focus on the adaptive minimal confidence region rule for multivariate initial bias truncation. This algorithm is similar to the Marginal Standard Error Rules that we call MSER. However, the MSER just is suitable for the univariate signal not multivariate systems. My work will focus on the multivariate. The MSER determines the truncation point (steady state point in this research) which minimizes the marginal confidence interval's the width about the mean of truncated sample (steady state mean). Thus, my work will do two things:

First, based on the MSER, I will try to get the volume of the confidence region of multivariate signals. This is very challenging and complex. 
Second, I will try to find a truncation point that minimizes the confidence region (volume) of the mean estimate of the observations after the truncation point. 


\section{References}

[1] K. Hoad, S. Robinson, and R. Davies, "Automating warm-up length estimation," Journal of the Operational Research Society, vol. 61, pp. 1389-1403, 2010.

[2] A. Marchetti, A. Ferramosca, and A. González, "Steady-state target optimization designs for integrating real-time optimization and model predictive control," Journal of Process Control, vol. 24, pp. 129-145, 2014.

[3] A. Mhamdi, W. Geffers, F. Flehmig, and W. Marquardt, "On-line optimization of MSF desalination plants," Desalination and Water Resources: Thermal Desalination Processes, vol. 1, pp. 136-162, 2010.

[4] N. Ricker and J. Lee, "Nonlinear modeling and state estimation for the Tennessee Eastman challenge process," Computers \& chemical engineering, vol. 19, pp. 983-1005, 1995.

[5] J. Chen and J. Howell, "A self-validating control system based approach to plant fault detection and diagnosis," Computers \& chemical engineering, vol. 25, pp. 337-358, 2001.

[6] M. Kim, S. H. Yoon, P. A. Domanski, and W. V. Payne, "Design of a steady-state detector for fault detection and diagnosis of a residential air conditioner," International journal of refrigeration, vol. 31, pp. 790-799, 2008.

[7] S. A. Bhat and D. N. Saraf, "Steady-state identification, gross error detection, and data reconciliation for industrial process units," Industrial \& engineering chemistry research, vol. 43, pp. 4323-4336, 2004.

[8] M. Schladt and B. Hu, "Soft sensors based on nonlinear steady-state data reconciliation in the process industry," Chemical Engineering and Processing: Process Intensification, vol. 46, pp. 1107-1115, 2007.

[9] S. Cao and R. R. Rhinehart, "An efficient method for on-line identification of steady state," Journal of Process Control, vol. 5, pp. 363-374, 1995.

[10] S. K. Mahuli, R. R. Rhinehart, and J. B. Riggs, "Experimental demonstration of non-linear model-based in-line control of pH," Journal of Process Control, vol. 2, pp. 145-153, 1992.

[11] J. Wu, S. Zhou, and X. Li, "Acoustic emission monitoring for ultrasonic cavitation based dispersion process," Journal of Manufacturing Science and Engineering, vol. 135, p. 031015, 2013.

[12] T. Jiang, B. Chen, X. He, and P. Stuart, "Application of steady-state detection method based on wavelet transform," Computers \& chemical engineering, vol. 27, pp. 569-578, 2003.

[13] H. Li, "A decoupling-based unified fault detection and diagnosis approach for packaged air conditioners," 2004.

[14] D. Aguado, A. Ferrer, A. Seco, and J. Ferrer, "Using Unfold-PCA for batch-to-batch startup process understanding and steady-state identification in a sequencing batch reactor," Journal of Chemometrics, vol. 22, pp. 81-90, 2008. 
[15] Y. Yao, C. Zhao, and F. Gao, "Batch-to-batch steady state identification based on variable correlation and mahalanobis distance," Industrial \& engineering chemistry research, vol. 48, pp. 11060-11070, 2009.

[16] S. Natarajan and R. R. Rhinehart, "Automated stopping criteria for neural network training," in American Control Conference, 1997. Proceedings of the 1997, 1997, pp. 24092413.

[17] W. W. Franklin, The Theoretical Foundation of the MSER Algorithm, 2009.

[18] S. Robinson, Simulation: the practice of model development and use: Palgrave Macmillan, 2014.

[19] S. Robinson, "A statistical process control approach to selecting a warm-up period for a discrete-event simulation," European Journal of Operational Research, vol. 176, pp. 332346, 2007.

[20] G. Gordon, 1969. System simulation. Prentice Hall, Englewood Cliffs, N.J.

[21] B. L. Nelson, "Statistical analysis of simulation results," Handbook of Industrial Engineering: Technology and Operations Management, Third Edition, pp. 2469-2495, 1992.

[22] M. D. Rossetti, Z. Li, and P. Qu, "Exploring exponentially weighted moving average control charts to determine the warm-up period," in Proceedings of the 37th conference on Winter simulation, 2005, pp. 771-780.

[23] K. P. White Jr, "An effective truncation heuristic for bias reduction in simulation output," Simulation, vol. 69, pp. 323-334, 1997.

[24] K. P. White Jr, M. J. Cobb, and S. C. Spratt, "A comparison of five steady-state truncation heuristics for simulation," in Proceedings of the 32nd conference on Winter simulation, 2000, pp. 755-760.

[25] K. Pawlikowski, "Steady-state simulation of queueing processes: survey of problems and solutions," ACM Computing Surveys (CSUR), vol. 22, pp. 123-170, 1990.

[26] E. K. Lada and J. R. Wilson, "A wavelet-based spectral procedure for steady-state simulation analysis," European Journal of Operational Research, vol. 174, pp. 1769-1801, 2006.

[27] D. H. Ockerman and D. Goldsman, "Student t-tests and compound tests to detect transients in simulated time series," European Journal of Operational Research, vol. 116, pp. 681691, 1999.

[28] P. Jackway and B. M. Desilva, "A methodology for initialisation bias reduction in computer simulation output," 1992.

[29] R. M. Bethea and R. R. Rhinehart, Applied engineering statistics vol. 121: CRC Press, 1991.

[30] W. Holly, R. Cook, and C. Crowe, "Reconciliation of mass flow rate measurements in a chemical extraction plant," The Canadian Journal of Chemical Engineering, vol. 67, pp. 595-601, 1989. 
[31] S. Narasimhan, C. S. Kao, and R. Mah, "Detecting changes of steady states using the mathematical theory of evidence," AIChE journal, vol. 33, pp. 1930-1932, 1987.

[32] E. L. Crow, F. A. Davis, and M. W. Maxfield, Statistics manual: with examples taken from ordnance development vol. 3369: Courier Corporation, 1960.

[33] J. Wu, Y. Chen, and S. Zhou, "Online detection of steady-state operation using a multiplechange-point model and exact Bayesian inference," IIE Transactions, vol. 48, pp. 599-613, 2016.

[34] J. Wu, Y. Chen, S. Zhou, and X. Li, "Online steady-state detection for process control using multiple change-point models and particle filters," IEEE Transactions on Automation Science and Engineering, vol. 13, pp. 688-700, 2016.

[35] Y. Hou, J. Wu, and Y. Chen, "Online Steady State Detection Based on Rao-Blackwellized Sequential Monte Carlo," Quality and Reliability Engineering International, vol. 32, pp. 2667-2683, 2016.

[36] P. R. Brown and R. R. Rhinehart, "Demonstration of a method for automated steady-state identification in multivariable systems," Hydrocarbon processing, vol. 79, pp. 79-83, 2000.

[37] J. D. Kelly and J. D. Hedengren, "A steady-state detection (SSD) algorithm to detect nonstationary drifts in processes," Journal of Process Control, vol. 23, pp. 326-331, 2013.

[38] Y. Yang and X. Li, "Ultrasonic cavitation based nanomanufacturing of bulk aluminum matrix nanocomposites," Journal of manufacturing science and engineering, vol. 129, pp. 497-501, 2007. 


\section{Appendix}

\section{Appendix A: Covariance of $\boldsymbol{X}_{f, t}$}

Based on the Eq.(3.2), we can get

$X_{f, t}=\lambda_{1} X_{t}+\left(1-\lambda_{1}\right) X_{f, t-1}$

$=\lambda_{1} X_{t}+\left(1-\lambda_{1}\right)\left[\lambda_{1} X_{t-1}+\left(1-\lambda_{1}\right) X_{f, t-2}\right]$

$=\lambda_{1} X_{t}+\lambda_{1}\left(1-\lambda_{1}\right) X_{t-1}+\cdots+\lambda_{1}\left(1-\lambda_{1}\right)^{t-2} X_{2}+\left(1-\lambda_{1}\right)^{t-1} X_{f, 1}$

$=\left\{\lambda_{1} X_{t}+\lambda_{1}\left(1-\lambda_{1}\right) X_{t-1}+\cdots+\lambda_{1}\left(1-\lambda_{1}\right)^{t-2} X_{2}\right\}+\left(1-\lambda_{1}\right)^{t-1} X_{f, 1}$

Since $X_{f, 1}=X_{1}$ and the covariance of $X_{t}$ is unchanged in the whole process, the covariance of $X_{f, t}$ can be derived as

$$
\begin{gathered}
\Sigma_{X_{f, t}}=\lambda_{1}^{2}\left(1+\left(1-\lambda_{1}\right)^{2}+\left(1-\lambda_{1}\right)^{4}+\cdots+\left(1-\lambda_{1}\right)^{2(t-2)}\right) \Sigma_{x}+\left(1-\lambda_{1}\right)^{2(t-1)} \Sigma_{x} \\
=\left[\frac{\lambda_{1}\left(1-\left(1-\lambda_{1}\right)^{2 t}\right)}{2-\lambda_{1}}+\left(1-\lambda_{1}\right)^{2(t-1)}\right] \Sigma_{x}
\end{gathered}
$$

As $t \rightarrow \infty$,

$$
\Sigma_{X_{f, t}}=\frac{\lambda_{1}}{2-\lambda_{1}} \sum_{x}
$$




\section{Appendix B: Expectation of $\widehat{\boldsymbol{\Sigma}}_{\boldsymbol{f}, t}$}

Based on the Eq. (3.2), we can get

$$
E\left(X_{f, t}\right)=\lambda_{1} E\left(X_{t}\right)+\left(1-\lambda_{1}\right) E\left(X_{f, t-1}\right)
$$

In the stationary state, the expectation of $X_{t}$ is unchanged. For simplicity, we assume $t_{0}$ is the steady state transition time. Denote the mean $E\left(X_{t}\right)=\mu$ for $t \geq t_{0}$, then

$$
\begin{aligned}
E\left(X_{f, t}\right)-\mu= & \lambda_{1} E\left(X_{t}\right)+\left(1-\lambda_{1}\right) E\left(X_{f, t-1}\right)-\mu=\lambda_{1} \mu+\left(1-\lambda_{1}\right) E\left(X_{f, t-1}\right)-\mu \\
& =\left(1-\lambda_{1}\right)\left(E\left(X_{f, t-1}\right)-\mu\right)=\left(1-\lambda_{1}\right)^{\left(t-t_{0}\right)}\left(E\left(X_{f, t_{0}}\right)-\mu\right)
\end{aligned}
$$

As $t \rightarrow \infty,\left(1-\lambda_{1}\right)^{\left(t-t_{0}\right)}\left(E\left(X_{f, t_{0}}\right)-\mu\right) \rightarrow 0$. Therefore

$$
E\left(X_{f, t}\right)=\mu=E\left(X_{t}\right)
$$

Based on Eq. (3.5), the expectation of $\hat{\Sigma}_{f, t}$ is derived as follows.

$$
\begin{gathered}
E\left(\widehat{\Sigma}_{f, t}\right)=\lambda_{2} E\left(\left(X_{t}-X_{f, t-1}\right)\left(X_{t}-X_{f, t-1}\right)^{T}\right)+\left(1-\lambda_{2}\right) E\left(\widehat{\Sigma}_{f, t-1}\right) \\
=\lambda_{2} E\left(\left(X_{t}-\mu+\mu-X_{f, t-1}\right)\left(X_{t}-\mu+\mu-X_{f, t-1}\right)^{T}\right)+\left(1-\lambda_{2}\right) E\left(\widehat{\Sigma}_{f, t-1}\right) \\
=\lambda_{2}\left(E\left[\left(X_{t}-\mu\right)\left(X_{t}-\mu\right)^{T}\right]+E\left[\left(X_{t}-\mu\right)\left(\mu-X_{f, t-1}\right)^{T}\right]+E\left[\left(\mu-X_{f, t-1}\right)\left(X_{t}-\mu\right)^{T}\right]\right. \\
\left.+E\left[\left(\mu-X_{f, t-1}\right)\left(\mu-X_{f, t-1}\right)^{T}\right]\right)+\left(1-\lambda_{2}\right) E\left(\hat{\Sigma}_{f, t-1}\right)
\end{gathered}
$$

Since $X_{t}$ and $X_{f, t-1}$ are independent, $E\left[\left(X_{t}-\mu\right)\left(\mu-X_{f, t-1}\right)^{T}\right]=E\left[\left(\mu-X_{f, t-1}\right)\left(X_{t}-\right.\right.$ $\left.\mu)^{T}\right]=0$. Meanwhile, $E\left[\left(X_{t}-\mu\right)\left(X_{t}-\mu\right)^{T}\right]=\Sigma_{x} \quad$ and $\quad E\left[\left(\mu-X_{f, t-1}\right)\left(\mu-X_{f, t-1}\right)^{T}\right]=$ $\Sigma_{X_{f, t-1}}=\Sigma_{X_{f, t}}$ (based on Eq.(3.4)). Therefore

$$
E\left(\widehat{\Sigma}_{f, t}\right)=\lambda_{2}\left(\Sigma_{x}+\Sigma_{X_{f, t}}\right)+\left(1-\lambda_{2}\right) E\left(\widehat{\Sigma}_{f, t-1}\right)
$$

Denote $C_{t}=\Sigma_{x}+\Sigma_{X_{f, t}}$. Suppose $t \gg t^{\prime} \gg t_{0}$, based on Eq.(3.4), $C_{t}=C_{t-1}=\cdots=C_{t^{\prime}+1}=$ $\frac{2}{2-\lambda_{1}} \sum_{x}$. When $t-t^{\prime} \rightarrow \infty$

$$
\begin{gathered}
E\left(\widehat{\Sigma}_{f, t}\right)=\lambda_{2} C_{t}+\lambda_{2}\left(1-\lambda_{2}\right) C_{t-1}+\cdots+\lambda_{2}\left(1-\lambda_{2}\right)^{t-t^{\prime}-1} C_{t^{\prime}+1}+\left(1-\lambda_{2}\right)^{t-t^{\prime}} E\left(\widehat{\Sigma}_{f, t^{\prime}}\right) \\
=\lambda_{2} \times \frac{2 \Sigma_{x}}{2-\lambda_{1}} \frac{\left(1-\left(1-\lambda_{2}\right)^{t-t^{\prime}}\right)}{1-\left(1-\lambda_{2}\right)}+\left(1-\lambda_{2}\right)^{t-t^{\prime}} E\left(\widehat{\Sigma}_{f, t^{\prime}}\right)=\frac{2 \Sigma_{x}}{2-\lambda_{1}}
\end{gathered}
$$




\section{Appendix C: Expectation of $\widehat{\boldsymbol{\Sigma}}_{s, t}$}

$$
\begin{gathered}
E\left(\hat{\Sigma}_{s, t}\right)=\lambda_{3} E\left(\left(X_{t}-X_{t-1}\right)\left(X_{t}-X_{t-1}\right)^{T}\right)+\left(1-\lambda_{3}\right) E\left(\hat{\Sigma}_{s, t-1}\right) \\
=\lambda_{3}\left(E\left(X_{t}-\mu\right)\left(X_{t}-\mu\right)^{T}+E\left(\mu-X_{t-1}\right)\left(\mu-X_{t-1}\right)^{T}\right)+\left(1-\lambda_{3}\right) E\left(\hat{\Sigma}_{s, t-1}\right)
\end{gathered}
$$

In the stationary period, $E\left(X_{t-1}\right)=E\left(X_{t}\right)=\mu$, therefore

$$
E\left(\hat{\Sigma}_{s, t}\right)=2 \lambda_{3} \Sigma_{x}+\left(1-\lambda_{3}\right) E\left(\hat{\Sigma}_{s, t-1}\right)
$$

Similar to the derivation of $E\left(\hat{\Sigma}_{f, t}\right)$, as $t \rightarrow \infty$,

$$
E\left(\widehat{\Sigma}_{s, t}\right)=2 \Sigma_{x}
$$




\section{Vita}

Honglun $\mathrm{Xu}$ received the bachelor degree in Petroleum Engineering from Northeast Petroleum University, Daqing, China in 2013 and the master degree in Petroleum and Natural Gas Engineering from China University of Petroleum (Beijing), China in 2016.

In 2016, he joined the doctoral program in Computational Science Program at the University of Texas at El Paso (UTEP), TX, USA. His research interests are focused on statistical modeling, process monitoring and quality control.

While pursuing his degree, he worked as a research assistant for the Department of Industrial Manufacturing and System Engineering and a teaching assistant for the Computational Science Program.

Contact Information : hxu3@miners.utep.edu

This thesis was typed by Honglun Xu.

Publications: Xu, H., Wu, J.*, Tseng, B., (2018), "An efficient method for on-line identification of steady state for multivariate systems", ASME 2018 13th International Manufacturing Science and Engineering Conference. 